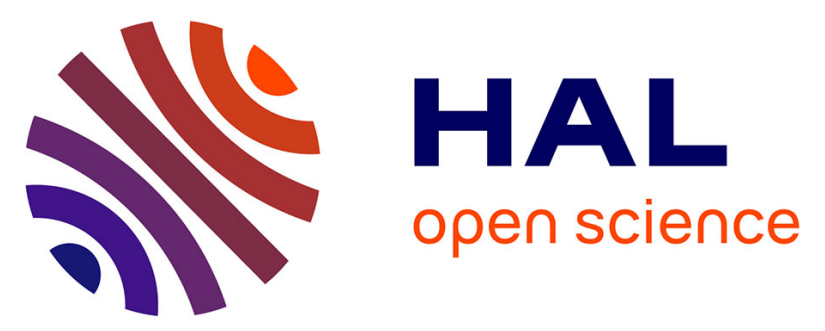

\title{
Fundamental Noise Limits and Sensitivity of Piezoelectrically Driven Magnetoelastic Cantilevers
}

Phillip Durdaut, Enrico Rubiola, Jean-Michel Friedt, Cai Müller, Benjamin Spetzler, Christine Kirchhof, Dirk Meyners, Eckhard Quandt, Franz Faupel, Jeffrey Mccord, et al.

\section{To cite this version:}

Phillip Durdaut, Enrico Rubiola, Jean-Michel Friedt, Cai Müller, Benjamin Spetzler, et al.. Fundamental Noise Limits and Sensitivity of Piezoelectrically Driven Magnetoelastic Cantilevers. Journal of Microelectromechanical Systems, 2020, 29 (5), pp.1347 - 1361. hal-02993766

\section{HAL Id: hal-02993766 https://hal.science/hal-02993766}

Submitted on 7 Nov 2020

HAL is a multi-disciplinary open access archive for the deposit and dissemination of scientific research documents, whether they are published or not. The documents may come from teaching and research institutions in France or abroad, or from public or private research centers.
L'archive ouverte pluridisciplinaire HAL, est destinée au dépôt et à la diffusion de documents scientifiques de niveau recherche, publiés ou non, émanant des établissements d'enseignement et de recherche français ou étrangers, des laboratoires publics ou privés. 


\title{
Fundamental Noise Limits and Sensitivity of Piezoelectrically Driven Magnetoelastic Cantilevers
}

\author{
Phillip Durdaut, Graduate Student Member, IEEE, Enrico Rubiola, Member, IEEE, Jean-Michel Friedt, Cai Müller, \\ Benjamin Spetzler, Christine Kirchhof, Dirk Meyners, Eckhard Quandt, Franz Faupel, Jeffrey McCord, Senior \\ Member, IEEE, Reinhard Knöchel, Life Fellow, IEEE, and Michael Höft, Senior Member, IEEE
}

\begin{abstract}
Magnetoelastic sensors for the detection of lowfrequency and low-amplitude magnetic fields are in the focus of research for more than $\mathbf{3 0}$ years. In order to minimize the limit of detection (LOD) of such sensor systems, it is of high importance to understand and to be able to quantify the relevant noise sources. In this contribution, cantilever-type electromechanical and magnetoelastic resonators, respectively, are comprehensively investigated and mathematically described not only with regard to their phase sensitivity but especially to the extent of the sensor-intrinsic phase noise. Both measurements and calculations reveal that the fundamental LOD is limited by additive phase noise due to thermal-mechanical noise of the resonator, i.e. by thermally induced random vibrations of the cantilever, and by thermal-electrical noise of the piezoelectric material. However, due to losses in the magnetic material parametric flicker phase noise arises, limiting the overall performance. In particular, it is shown that the LOD is virtually independent of the magnetic sensitivity but is solely determined by the magnetic losses. Instead of the sensitivity, the magnetic losses, represented by the material's effective complex permeability, should be considered as the most important parameter for the further improvement of such sensors in the future. This implication is not only valid for magnetoelastic cantilevers but also applies to any type of magnetoelastic resonator.
\end{abstract}

Index Terms-Cantilever, delta-E effect, flicker phase noise, limit of detection, magnetic field sensor, magnetic noise, magnetoelastic sensor, phase noise, phase sensitivity, resonator, thermal noise

\section{INTRODUCTION}

In 1989 Brendel et al. reported on a parasitic influence of magnetic fields on the oscillation frequency of quartz crystal oscillators which could be explained by magnetically induced deformations in the partly ferromagnetic springs used to hold the quartz plate [1], [2]. Since then, various micromechanical sensors based on the $\Delta \mathrm{E}$ effect (Sec. II-A) have been presented, whose mechanical properties depend on an external

P. Durdaut, R. Knöchel and M. Höft are with the Chair of Microwave Engineering, Institute of Electrical Engineering and Information Technology, Kiel University, 24143 Kiel, Germany.

E. Rubiola and J.-M. Friedt are with the FEMTO-ST Institute, Department of Time and Frequency, Université de Bourgogne Franche-Comté (UBFC), and CNRS, ENSMM, 26 Rue de l'Épitaphe, 25000 Besançon, France. E. Rubiola is also with the Physics Metrology Division, Istituto Nazionale di Ricerca Metrologica (INRiM), Strada Delle Cacce 91, 10135 Torino, Italy.

C. Müller and J. McCord are with the Chair of Nanoscale Magnetic Materials and Magnetic Domains, Institute for Materials Science, Kiel University, 24143 Kiel, Germany.

C. Kirchhof, D. Meyners, E. Quandt are with the Chair of Inorganic Functional Materials, Institute for Materials Science, Kiel University, 24143 Kiel, Germany.

B. Spetzler and F. Faupel are with the Chair for Multicomponent Materials, Institute for Materials Science, Kiel University, 24143 Kiel, Germany. magnetic field through interaction with a magnetostrictive layer. Although realizations in the form of highly sensitive magnetoelastic surface acoustic wave delay lines were also presented [3]-[9], magnetoelastic sensors are most commonly based on resonant structures [10]-[18], especially cantilevers [19]-[23], with resonance frequencies in the range between $550 \mathrm{~Hz}$ and $226 \mathrm{MHz}$.

Besides properties like e.g. dynamic range and frequency bandwidth, the limit of detection (LOD), frequently also referred to as detectivity or equivalent magnetic noise floor, is often considered as one of the most important figures of merit of a magnetic field sensor. Similar to a signal-to-noise ratio (SNR), the LOD is determined by both the sensor's signal, i.e. the sensitivity, as well as by the sensor's noise properties. In the existing articles reporting about magnetoelastic resonators, the focus has mostly been on modified sensor structures and their properties with an emphasis on enhancing the effect, i.e. the detuning of the sensing resonator. Although articles reported on measured values for the limit of detection in the microtesla [10], [12], [19], nanotesla [13], [16], [18], [20], [21], and even in the picotesla [11], [15], [22] range, the physical causes for noise in magnetoelastic magnetic field sensors based on the $\Delta \mathrm{E}$ effect have not been investigated and described yet.

In previous studies, we focused on thermal noise of magnetoelectric cantilevers in passive mode [24], on the realization and analysis of low-noise preamplifiers for such sensors [25], on noise contributions of the readout electronics [26], [27] and the suppression of the local oscillator's phase noise in active mode magnetoelastic sensor systems [28]. Based on this, in this paper the influence of the sensor's thermal noise on the phase noise is analyzed both metrologically and analytically. In addition, the impact of losses in the magnetic material on the phase noise characteristics, and thus on the overall sensor performance is shown.

This paper is organized as follows: Sec. II introduces the sensor principle and the actual structure of the magnetoelastic cantilever under investigation. Based on the dynamics of resonant mechanical structures, expressions for the various sensitivities are derived, yielding the overall phase sensitivity. In addition, both an electrical equivalent circuit of the sensor covering for the various loss mechanisms as well a phase detecting readout system is presented. In comparison to previous studies the latter has been modified in order to allow for the neutralization of the sensor's parasitic static capacitance responsible for asymmetric transmission characteristics and a 
reduced sensitivity. Based on the sensor's loss mechanisms, expressions for thermally induced phase noise are derived and verified by measurements in Sec. III. Additional flicker phase noise clearly related to the losses in the magnetic material are traced back to fluctuations of the magnetization. Based on the fluctuation-dissipation theorem analytical expressions for the magnetically induced phase noise as well as for the resulting limit of detection are deduced. This article finishes with a summary of the findings in Sec. IV.

\section{Magnetoelastic Sensor System}

\section{A. $\Delta E$ effect}

The Young's modulus of any material is defined by the ratio between stress $\sigma$ and elastic strain $\varepsilon_{\mathrm{el}}$ that is measured in the direction parallel to the applied stress [29]. However, for magnetic materials the relation $E_{\mathrm{sat}}=\sigma / \varepsilon_{\mathrm{el}}$ is only valid for magnetically saturated specimen [29]. In the general case, the problem has to be treated using tensors. As a consequence of the magnetostrictive effect, an additional magnetoelastic strain $\varepsilon_{\text {mel }}$ occurs in magnetic materials [30, p. 270]. According to $\Delta \varepsilon_{\text {mel }}=d^{\mathrm{m}} \Delta H$ (for positive magnetostriction) the magnetoelastic strain directly changes with the magnetic field $H$ and proportionally to the piezomagnetic constant $d^{\mathrm{m}}$ [31] if $\varepsilon_{\text {mel }}$ is linearized around a certain magnetic operating point $H_{\text {bias }}$. Considering both types of elastic strain, the resulting Young's modulus [30, p. 270]

$$
E(H)=\frac{\sigma}{\varepsilon_{\mathrm{el}}+\varepsilon_{\mathrm{mel}}(H)} \leq E_{\mathrm{sat}}
$$

depends on the magnetic field $H$ and is always lower than the Young's modulus of the same material in magnetic saturation. The magnetically induced change of the Young's modulus in the normalized form

$$
\Delta \mathrm{E} \text { effect } \equiv \frac{\Delta E}{E}=\frac{E_{\mathrm{sat}}-E}{E}=\frac{\varepsilon_{\mathrm{mel}}(H)}{\varepsilon_{\mathrm{el}}}
$$

is known as the $\Delta \mathrm{E}$ effect [31]. In the literature values for $\Delta E / E$ as high as approximately $700 \%$ for alloys of terbiumdysprosium (TbDy) [31] and approximately $30 \%$ for an alloy of iron-cobalt-silicon-boron $\left(\left(\mathrm{Fe}_{90} \mathrm{Co}_{10}\right)_{78} \mathrm{Si}_{12} \mathrm{~B}_{10}\right)$ [32] that is used in this work, respectively, are reported. It should be noted, however, that very large magnetic fields are required to change the Young's modulus in terbium-dysprosium by such a large value. With regard to the use of magnetostrictive materials for sensor applications, it is rather important how strong $E$ is changed by a low amplitude magnetic measurement signal $B_{\mathrm{x}}=\mu_{0} H_{\mathrm{x}}$ in a certain magnetic operating point $B_{\text {bias }}=\mu_{0} H_{\text {bias }}$, thus requiring materials with large piezomagnetic constants $d^{\mathrm{m}}$. An overview of piezomagnetic coefficients of various materials can be found in [33]. For $\left(\mathrm{Fe}_{90} \mathrm{Co}_{10}\right)_{78} \mathrm{Si}_{12} \mathrm{~B}_{10}$ a value of $60 \mathrm{~nm} / \mathrm{A}$ is reported that is only exceeded by $\left(\mathrm{Fe}_{90} \mathrm{Ga}_{19}\right)_{88} \mathrm{~B}{ }_{12}$ with a value of $151 \mathrm{~nm} / \mathrm{A}$.

\section{B. Magnetoelastic sensor}

The magnetoelastic sensor used for the investigations in this contribution as depicted in Fig. 1 is based on a polysilicon cantilever of $3 \mathrm{~mm}$ length, $1 \mathrm{~mm}$ width and $50 \mu \mathrm{m}$

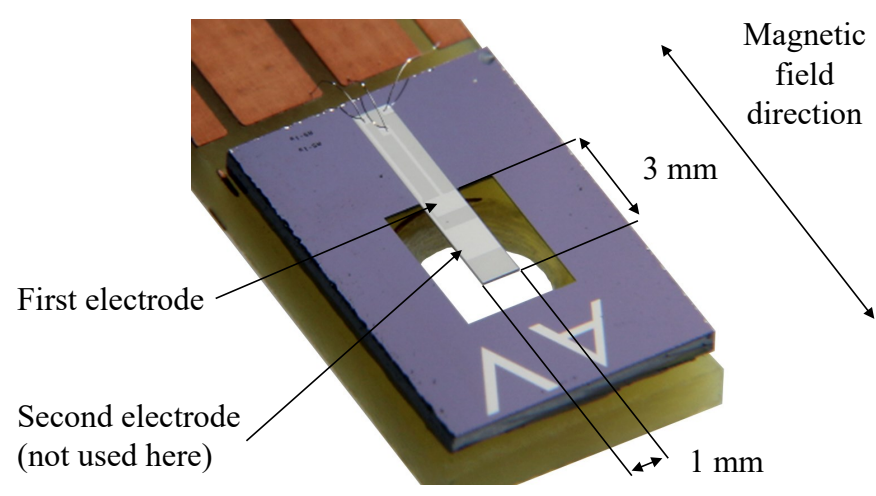

Fig. 1: Photograph of the utilized cantilever-type magnetoelastic sensor with a size of $3 \mathrm{~mm} \times 1 \mathrm{~mm}$ mounted to a carrier PCB. The magnetic flux densities $B_{\text {bias }}$ and $B_{\mathrm{x}}$ are applied along the long mechanical axis of the cantilever.

thickness. The lower side is coated with $2 \mu \mathrm{m}$ of soft magnetic amorphous metal $\left(\left(\mathrm{Fe}_{90} \mathrm{Co}_{10}\right)_{78} \mathrm{Si}_{12} \mathrm{~B}_{10}\right.$, magnetic easy axis perpendicular to the cantilever's long axis), and $2 \mu \mathrm{m}$ of aluminum-nitride (AlN) piezoelectric material [34] are deposited on the cantilever's top. Details about the MEMS fabrication process can be found in [22]. In addition, the sensor offers two independent types of electrodes (see Fig. 1) that form plate capacitors with the piezoelectric AlN being the dielectric material. The investigations in this contribution focus on the first bending mode for which the first electrode performs best [35].

\section{Resonance detuning and magnetic sensitivity}

The resonance frequency of a composite cantilever with $N$ layers is given by [22]

$$
f_{\mathrm{res}}=\frac{1}{2 \pi} \frac{\lambda^{2}}{l^{2}} \cdot \sqrt{\frac{\sum_{n=1}^{N} E_{n} J_{n}}{\sum_{n=1}^{N} m_{n}}},
$$

where $\lambda=1.875$ is the eigenvalue of the first characteristic bending mode, $l=3 \mathrm{~mm}$ is the free-standing length of the cantilever, and $E_{n}$ is the Young's modulus, $J_{n}$ is the second moment of area, and $m_{n}$ is the mass per unit length of the $n$ th layer, respectively. Thus, the sensor's resonance frequency is proportional to the square root of the composite's effective Young's modulus $E_{\text {eff }}$

$$
f_{\text {res }}\left(B_{\text {bias }}, B_{\mathrm{x}}\right) \propto \sqrt{E_{\text {eff }}\left(B_{\text {bias }}, B_{\mathrm{x}}\right)}
$$

that depends on an external magnetic field, i.e. on the bias flux density $B_{\text {bias }}$ and on the bias flux density of the measurement signal to be detected $B_{\mathrm{x}}$ (both applied along the long mechanical axis of the cantilever). As visible from a measurement in Fig. 2a, the cantilever's resonance frequency depends on the external magnetic field and changes particularly strong around $B_{\text {bias }}= \pm 0.65 \mathrm{mT}$. The corresponding slope is known as the 


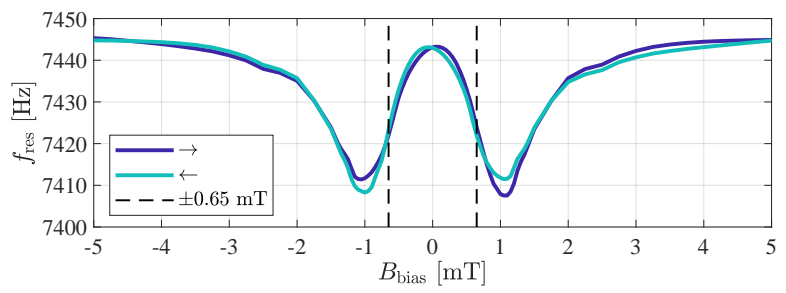

(a) Resonance frequency $f_{\text {res }}$ as a function of the external magnetic bias flux density $B_{\text {bias }}$

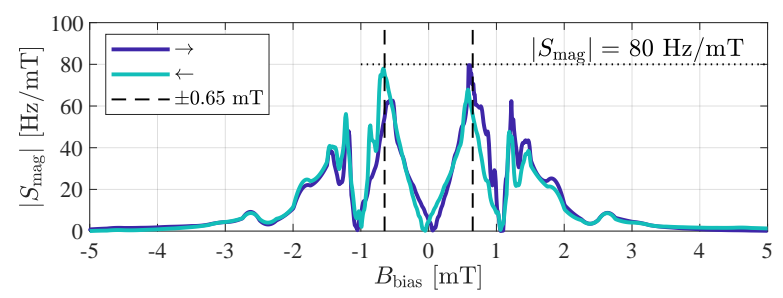

(b) Magnetic sensitivity $S_{\mathrm{mag}}$ as a function of the external magnetic bias flux density $B_{\text {bias }}$

Fig. 2: Detuning of the magnetoelastic cantilever's resonance frequency with an external magnetic flux density (a). High magnetic sensitivities (b) are reached where the resonance frequency changes particularly strong with an external magnetic flux density, i.e. at $B_{\text {bias }}= \pm 0.65 \mathrm{mT}$ (vertical dashed lines).

magnetic sensitivity towards low amplitude and low frequency magnetic measurement signals $B_{\mathrm{x}}$

$$
S_{\text {mag }}\left(B_{\text {bias }}\right)=\frac{\partial f_{\text {res }}}{\partial B_{\mathrm{x}}}
$$

for which a maximum value of $\left|S_{\mathrm{mag}}\right|=80 \mathrm{~Hz} / \mathrm{mT}$ is reached for the sensor under investigation (Fig. 2b). That value is often given normalized to $f_{\text {res }}$ in saturation (here $7445 \mathrm{~Hz}$ ), thus leading to a normalized magnetic sensitivity of $\left|S_{\mathrm{mag}}\right| \approx 1.07 \% \mathrm{f}_{\mathrm{res}} / \mathrm{mT}$ which is a typical value for thinfilm magnetoelastic resonators [10], [11], [15], [21], [22].

In addition to the magnetic sensitivity determinable based on the magnetic field dependent resonance frequency, the measured characteristic in Fig. 2a reveals further insights into the sensor's magnetic behavior. The different results depending on the sweep direction of the applied magnetic bias flux density are an indication of magnetic hysteresis. This reflects that the magnetic state of the piezomagnetic layer depends on the history of the applied magnetic bias flux density. During a sweep of the applied magnetic bias flux density the magnetic domain state changes, rearranging into different domain states at various points of the magnetization loop. These magnetic domain states and their dependence on the magnetic history of the applied magnetic bias flux density can be rather complex [36] and strongly influence the magnetic reversal process. Depending on the field amplitude and orientation magnetic domain behavior will change. It is often accompanied by nucleation, annihilation, and irreversible movement of magnetic domain walls. Similar magnetoelectric cantilever structures revealed magnetic Barkhausen noise contributions [37] due to magnetic domain reorganization processes during reversal. Especially, the variable domain structures also lead to variations in the effective sensitivity [37] and thus magnetic domain alterations relate to magnetic noise in such sensors [38]. Magnetic hysteresis losses are moreover linked to energy conversion into heat [39] and, thus, as discussed further below, correspond to additional noise contributions.

Contrary to all measurements for this investigation performed in an ultra-high magnetic field shielding mu-metal cylinder (Aaronia AG, ZG1), the magnetic operating point of the sensor can change due to ambient static fields outside magnetic shielding. For the sensor under investigation the impact of e.g. earth's magnetic field with magnetic flux densities between $25 \mu \mathrm{T}$ and $60 \mu \mathrm{T}$ [40, p. 43] is relatively low. To compensate for such external fields, self-regulating operating point stabilization approaches such as those already developed for giant magnetoimpedance (GMI) sensors [41] can be used. However, an operating point stabilization, i.e. providing a very stable and adjustable magnetic bias field, is not trivial because the required electronics also introduce additional noise [42]. Furthermore, the piezomagnetic layer of this sensor exhibits a uniaxial magnetic anisotropy. The orientation of the anisotropy favors the orientation of the magnetic field along the long axis of the cantilever. While additionally biasing the sensor in other directions proves useful in special cases [43], deviations of $B_{\text {bias }}$ from the long axis in this case generally deteriorates the sensitivity due to the quadratic nature of the magnetoelastic effect. This affects the sensor's linearity, i.e. the dynamic range. As discussed above, a different alignment of the magnetic field will lead to a different magnetic domain structure and thus a different contribution to the magnetic noise, leading to a change in LOD. These effects are currently under investigation.

\section{Mechanical behavior and electrical sensitivity}

Generally, the mechanical behavior of a resonant cantilever with the quality factor $Q$ can be described by the unitless frequency response of a simple damped harmonic oscillator [44, pp. 427] [45]

$$
G(f)=\frac{1}{1-\left(\frac{f}{f_{\text {res }}}\right)^{2}+j \frac{f}{f_{\text {res } Q}}}=|G(f)| \cdot \exp (j \gamma(f))
$$

with the magnitude frequency response

$$
|G(f)|=\frac{1}{\sqrt{\left(1-\left(\frac{f}{f_{\text {res }}}\right)^{2}\right)^{2}+\left(\frac{f}{f_{\text {res }}}\right)^{2}}}
$$

and the phase response

$$
\gamma(f)=\arctan \left(\frac{-\frac{f}{f_{\text {res }}}}{Q\left(1-\left(\frac{f}{f_{\text {res }}}\right)^{2}\right)}\right) .
$$

Eq. (8) describes the relation between phase and frequency where the slope of $\gamma(f)$ at $f_{\text {res }}$ yields the well-known expression

$$
S_{\text {elec }}=\left.\frac{\mathrm{d} \gamma(f)}{\mathrm{d} f}\right|_{f=f_{\mathrm{res}}}=-\frac{2 Q}{f_{\text {res }}}
$$


which, in the following, is referred to as the electrical sensitivity $S_{\text {elec }}$ of a resonant sensor in units of $\mathrm{rad} / \mathrm{Hz}$ [46].

\section{E. Dynamic frequency response and overall phase sensitivity}

The higher the quality factor $Q$ of a resonant sensor, the narrower the bandwidth of the characteristic bandpass behavior. Assuming a magnetic measurement signal $B_{\mathrm{x}}(t)=\hat{B}_{\mathrm{x}} \cos \left(2 \pi f_{\mathrm{x}} t\right)$, the sensor's response to such a signal with the frequency $f_{\mathrm{x}}$ can be determined by replacing $f$ with $f_{\text {res }} \pm f_{\mathrm{x}}[45]$ in Eq. (6)

$$
\begin{aligned}
G\left(f_{\text {res }} \pm f_{\mathrm{x}}\right) & =\frac{-j Q}{1 \pm \frac{f_{\mathrm{x}}}{f_{\text {res }}}+j \frac{Q f_{\mathrm{x}}^{2}}{f_{\text {res }}^{2}} \pm j \frac{2 Q f_{\mathrm{x}}}{f_{\text {res }}}} \\
& \approx \frac{-j Q}{1 \pm j \frac{2 Q f_{\mathrm{x}}}{f_{\text {res }}}} .
\end{aligned}
$$

Based on that result, an expression for the unitless dynamic sensitivity $S_{\mathrm{dyn}}$ can be deduced

$$
\begin{aligned}
S_{\mathrm{dyn}}\left(f_{\mathrm{x}}\right) & =\frac{G\left(f_{\text {res }} \pm f_{\mathrm{x}}\right)}{G\left(f_{\text {res }}\right)} \\
\approx & \frac{1}{\sqrt{1+\left(\frac{f_{\mathrm{x}}}{f_{\mathrm{c}}}\right)^{2}}} \cdot \exp \left(j \arctan \left(-\frac{f_{\mathrm{x}}}{f_{\mathrm{c}}}\right)\right)
\end{aligned}
$$

which exhibits the characteristic of a simple first-order lowpass filter with a cutoff frequency of $f_{\mathrm{c}}=f_{\text {res }} /(2 Q)$. This result agrees with theoretical expectations in [47] and with measurement results in [48]. Thus, the overall phase sensitivity of the resonant sensor in units of $\mathrm{rad} / \mathrm{T}$ yields

$$
S_{\mathrm{PM}}\left(B_{\text {bias }}, f_{\mathrm{x}}\right)=S_{\mathrm{mag}}\left(B_{\text {bias }}\right) \cdot S_{\text {elec }} \cdot S_{\text {dyn }}\left(f_{\mathrm{x}}\right) .
$$

\section{F. Electrical equivalent circuit and loss mechanisms}

According to the physical structure of the electromechanical resonator it can be described by an electrical equivalent circuit as depicted in the dashed box in Fig. 5 whose element's values can be determined utilizing a conventional impedance analyzer [22], [24], [26]. Based on electromechanical analogies [49] the mechanical structure's resonant behavior is taken into account by an electrical series resonant circuit with the impedance $Z_{\mathrm{r}}=R_{\mathrm{r}}+R_{\mathrm{mag}}+j \omega L_{\mathrm{r}}+1 /\left(j \omega C_{\mathrm{r}}\right)$ where $\omega=2 \pi f$ is the angular frequency. Due to the magnetically induced changes of the resonance frequency $f_{\text {res }}=1 /\left(2 \pi \sqrt{L_{\mathrm{r}} C_{\mathrm{r}}}\right)$, both the inductance $L_{\mathrm{r}}$ as well as the capacitance $C_{\mathrm{r}}$ change with the magnetic field. In parallel to the series resonant circuit the static capacitance due to the electrodes surrounding the piezoelectric material is considered by an additional capacitor with a capacitance of $C_{\mathrm{ME}}=44 \mathrm{pF}$ for the sensor under investigation.

A piezoelectric cantilever-type magnetoelastic sensor comprises several loss mechanisms that, according to the fluctuation-dissipation theorem, correspond with fluctuations, i.e. with noise. Generally, such losses can be taken into account in an electrical equivalent circuit in the form of dissipative elements, i.e. by resistors.

The predominant loss mechanism of micromechanical cantilevers under atmospheric pressure is air damping, commonly

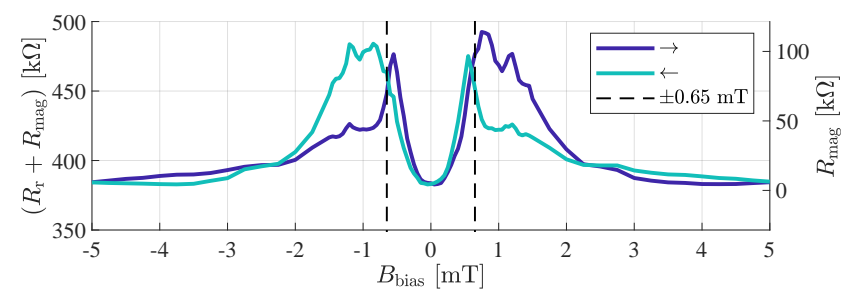

(a) Loss-representing values of the resistances $R_{\mathrm{r}}$ and $R_{\mathrm{mag}}$ as a function of the external magnetic bias flux density $B_{\text {bias }}$

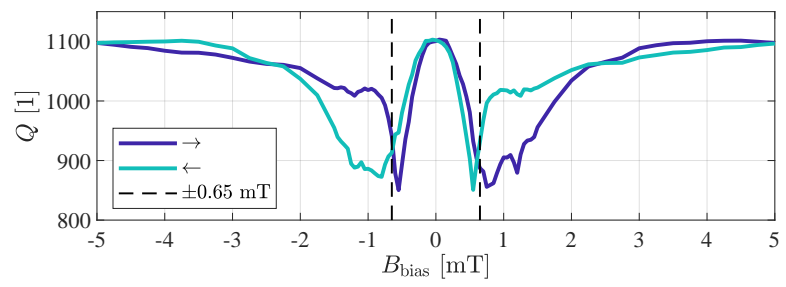

(b) Quality factor $Q$ as a function of the external magnetic bias flux density $B_{\text {bias }}$

Fig. 3: Losses, represented by the resistances $R_{\mathrm{r}}$ and $R_{\mathrm{mag}}$ (a), and corresponding quality factor $Q$ (b) as a function of the external magnetic bias flux density $B_{\text {bias }}$ measured for an amplitude of the electrical excitation signal of $\hat{V}_{\text {ex }}=100 \mathrm{mV}$. The losses are particularly high for bias flux densities that also lead to high magnetic sensitivities $S_{\text {mag }}$ (illustrated by the vertical dashed lines, compare also Fig. 2b) whereas highest values for $Q$ are obtained in magnetic saturation.

referred to as viscous damping. In addition, e.g. thermoelastic friction intrinsic to the solid structure, support losses, surface losses, and mounting losses (compare [50] for a more detailed analysis for a cantilever like the one investigated here or e.g. [51] for a general overview) may further attenuate the cantilever's deflection, also expressed by its quality factor $Q$. In the electrical equivalent circuit model (dashed box in Fig. 5) these losses are taken into account by the resistance $R_{\mathrm{r}}$.

For the special case of a magnetoelastic cantilever, additional losses occur as a function of its magnetic state, i.e. as a function of the external magnetic bias flux density $B_{\text {bias }}$ which are considered as an additional resistance $R_{\text {mag }}$. As measurement results in Fig. 3a reveal, these losses are particularly high for bias flux densities that also lead to high magnetic sensitivities $S_{\mathrm{mag}}$ (illustrated by the vertical dashed lines, compare also Fig. 2b). Conversely, this means that the overall quality factor

$$
Q\left(B_{\text {bias }}\right)=\frac{1}{R_{\mathrm{r}}+R_{\text {mag }}\left(B_{\text {bias }}\right)} \sqrt{\frac{L_{\mathrm{r}}\left(B_{\text {bias }}\right)}{C_{\mathrm{r}}\left(B_{\text {bias }}\right)}}
$$

is also a function of the bias flux density and that $Q$ is lower in the vicinity of the sensor's operating point (here $B_{\text {bias }}= \pm 0.65 \mathrm{mT}$ ) than in magnetic saturation (Fig. 3b). Results of a similar series of measurements in Fig. 4, but in dependence of the electrical excitation amplitude $V_{\text {ex }}$, clearly confirm the influence of the magnetic state on the losses and on the quality factor, respectively. In fact, the magnetic losses 


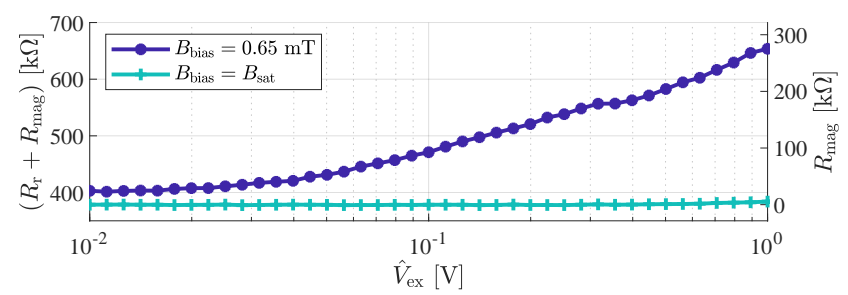

(a) Loss-representing values of the resistances $R_{\mathrm{r}}$ and $R_{\operatorname{mag}}$ as a function of the excitation amplitude $\hat{V}_{\text {ex }}$

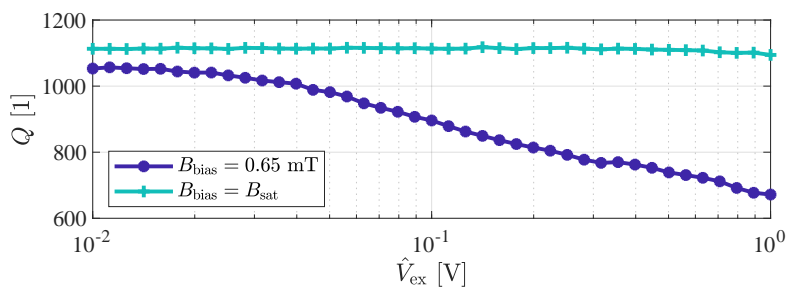

(b) Quality factor $Q$ as a function of the excitation amplitude $\hat{V}_{\text {ex }}$

Fig. 4: Losses, represented by the resistances $R_{\mathrm{r}}$ and $R_{\mathrm{mag}}$ (a), and corresponding quality factor $Q$ (b) as a function of the electrical excitation signal's amplitude $\hat{V}_{\text {ex }}$. If the sensor is not magnetically saturated the magnetic losses distinctly increase with the excitation amplitude due to dynamic magnetic hysteresis losses.

distinctly increase with higher excitation amplitudes in case the magnetic material is not in saturation.

As already hypothesized in [22], these additional losses can be explained by magnetic hysteresis losses that occur from the periodic bending of the cantilever which, in turn, lead to changes in the magnetization due to the inverse magnetostrictive effect, also referred to as Villari effect [52]. Dynamic magnetic hysteresis losses imply irreversible mechanisms due to domain activity that lead to energy dissipation in the form of heat during each cycle of periodic changes of the magnetization [53], [54]. Amongst other loss mechanisms related to the magnetic material like e.g. eddy current losses, the hysteresis losses are considered by the imaginary part $\mu_{\mathrm{r}}^{\prime \prime}$ of the magnetic material's relative permeability $\mu_{\mathrm{r}}=\mu_{\mathrm{r}}^{\prime}-j \mu_{\mathrm{r}}^{\prime \prime}$ [54].

Further losses are affiliated to the sensor's plate capacitor, i.e. to the piezoelectric material. These dielectric losses are considered by the loss tangent $\tan \delta_{\mathrm{ME}}$ with reported values for thin-film piezoelectric materials as low as e.g. 2.5 . $10^{-4}$ (aluminium-nitride, AlN) [55], $1.3 \cdot 10^{-3}$ (aluminiumscandium-nitride, $\mathrm{AlScN}$ ) [56], and $4 \cdot 10^{-3}$ (lead-zirconatetitanate, PZT) [57]. The sensor under investigation exhibits a value of $\tan \delta_{\mathrm{ME}}=5 \cdot 10^{-3}$, thus resulting in a resistance $R_{\mathrm{ME}}=\left(\tan \delta_{\mathrm{ME}} \omega C_{\mathrm{ME}}\right)^{-1}$ in parallel to the static capacitance $C_{\mathrm{ME}}$ and with a value in the vicinity of the resonance frequency of approximately $100 \mathrm{M} \Omega$. With a corresponding conductance $1 / R_{\mathrm{ME}}$ in the nanosiemens range its influence is usually negligible. However, in Sec. III it will be shown that the noise associated with these losses might degrade the sensor's performance under certain circumstances.

\section{G. Readout structure}

For sensor operation, i.e. for the reconstruction of a magnetic measurement signal $B_{\mathrm{x}}(t)$ due to an induced detuning of the resonator an electrical readout system as depicted in Fig. 5 is utilized. The basic principle is based on a resonant excitation of the sensor with a voltage signal $v_{\mathrm{ex}}(t)=\hat{V}_{\mathrm{ex}} \cos \left(2 \pi f_{\mathrm{ex}} t\right)$ with $f_{\text {ex }}=f_{\text {res }}$ (first bending mode) leading to a magnetically modulated current through the sensor $i_{\text {sensor }}(t)$ that, in turn, is transformed into a proportional voltage signal $v_{\text {sensor }}(t)$ utilizing a transimpedance amplifier and subsequent phase demodulation. For all measurements a low-noise JFET charge amplifier [25] with a feedback capacitance of $C_{\mathrm{f}}=30 \mathrm{pF}$ and a feedback resistance of $R_{\mathrm{f}}=5 \mathrm{G} \Omega$ is utilized whose transimpedance in the vicinity of $f_{\text {res }}$, i.e. far above the amplifier's lower cutoff frequency $\left(2 \pi R_{\mathrm{f}} C_{\mathrm{f}}\right)^{-1} \approx 1 \mathrm{~Hz}$, is given by

$$
T(f)=\frac{V_{\text {sensor }}(f)}{I_{\text {sensor }}(f)}=-\frac{1}{j 2 \pi f C_{\mathrm{f}}} .
$$

However, as already mentioned above, this type of electromechanical sensor has an additional static capacitance $C_{\mathrm{ME}}$ due to its electrodes that, with regard to the electrical equivalent circuit, appears in parallel to the series resonant circuit with the impedance $Z_{\mathrm{r}}$ leading to an overall admittance of the electromechanical sensor of

$$
Y_{\text {sensor }}=\frac{1}{Z_{\mathrm{r}}}+\frac{1}{R_{\mathrm{ME}}}+j \omega C_{\mathrm{ME}}
$$

As a consequence of the additional static capacitance $C_{\mathrm{ME}}$, a parallel resonance (also referred to as antiresonance) with a frequency $f_{\text {ares }}=f_{\text {res }} \sqrt{1+C_{\mathrm{r}} / C_{\mathrm{ME}}}$ slightly above $f_{\text {res }}$ [58, p. 204] appears which distorts the line shape of both the magnitude (Fig. 6a) and the phase (Fig. 6b) of $Y_{\text {sensor }}$ compared to that of a bare resonator described by $G(f)$ (Eq. (6)). As a result, the electrical sensitivity resulting by differentiation of the admittance's phase is smaller than stated in Eq. (9) (Fig. 6d). The Nyquist plot in Fig. 6c illustrates that effect by means of the phasor of the excitation signal, i.e. the carrier signal (gray lines), and by the highlighted resonance frequencies (red crosses) of the slightly detuned resonator. Although the effect is not as pronounced for the sensor under investigation due to its comparatively low static capacitance of only $C_{\mathrm{ME}}=44 \mathrm{pF}$, one can easily imagine that the larger the sensor's static capacitance (shift of the locus curve to the top), the lower the resulting phase modulation. This is the reason why various methods for neutralizing the sensor's static capacitance have been reported [59]-[62], especially for large capacitance sensors. On the contrary, for this contribution, a neutralization is performed for symmetrizing the sensor's behavior. Thus, the admittance can be described by the frequency response of a simple harmonic oscillator $G(f)$ (compare Fig. 6a and Fig. 6b) which simplifies the noise considerations in the following section.

In the actual system as depicted in Fig. 5 the neutralization is perceived by a second branch that contains a trimming capacitor with a capacitance of $C_{\mathrm{n}} \approx C_{\mathrm{ME}}$ and an identical charge amplifier as in the sensor branch. When neglecting the 


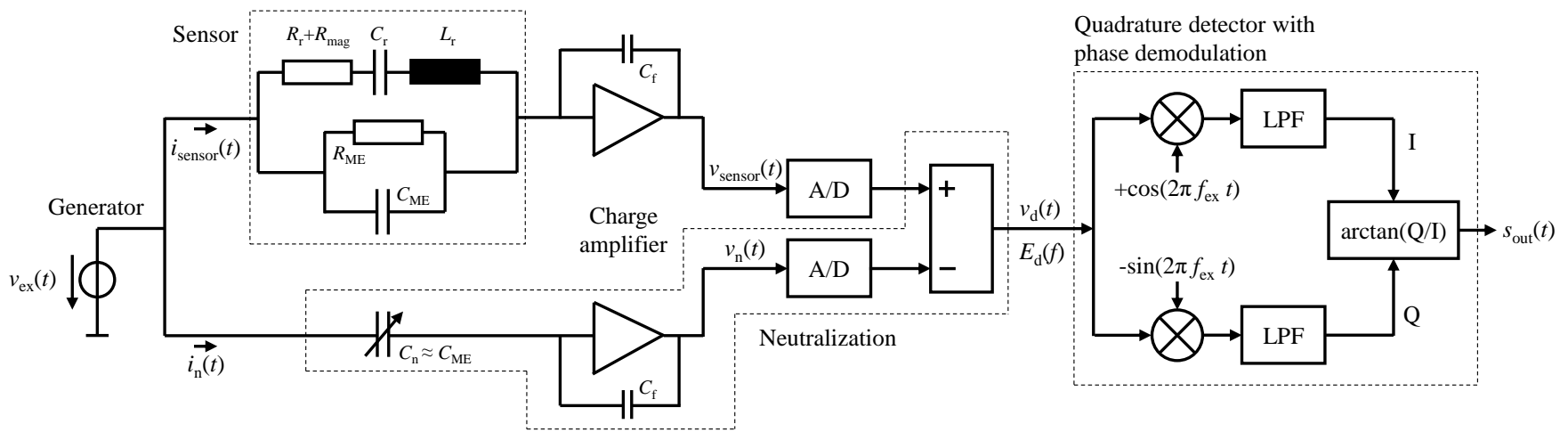

Fig. 5: Electrical readout system for the reconstruction of a magnetic measurement signal due to an induced detuning of the resonant sensor. The sensor (upper branch) is driven at its resonance frequency by an electrical excitation signal $v_{\text {ex }}(t)$, leading to a magnetically modulated voltage signal $v_{\text {sensor }}(t)$ at the output of the subsequent charge amplifier. An additional signal branch together with a subtractor is used to neutralize the parasitic effect of the sensor's static capacitance. The phase demodulation is performed in the digital domain by means of a conventional quadrature detector.

influence of the dielectric losses $\left(R_{\mathrm{ME}}\right)$ on the sensor signal $v_{\text {sensor }}(t)$ (see above) its amplitude spectrum yields

$$
\begin{aligned}
V_{\text {sensor }}(f) & =T I_{\text {sensor }}(f)=T V_{\text {ex }} Y_{\text {sensor }}(f) \\
& =T V_{\text {ex }}\left(\frac{1}{Z_{\mathrm{r}}(f)}+j 2 \pi f C_{\mathrm{ME}}\right) .
\end{aligned}
$$

Similarly, the amplitude spectrum at the output of the second charge amplifier is given by

$$
V_{\mathrm{n}}(f)=T I_{\mathrm{n}}(f)=T V_{\mathrm{ex}} j 2 \pi f C_{\mathrm{ME}},
$$

thus resulting in an amplitude spectrum of the differential signal $v_{\mathrm{d}}(t)$

$$
V_{\mathrm{d}}(f)=V_{\text {sensor }}(f)-V_{\mathrm{n}}(f)=\frac{T V_{\mathrm{ex}}}{Z_{\mathrm{r}}(f)}
$$

in which the parasitic influence of the static capacitance $C_{\mathrm{ME}}$ is suppressed. For a resonant excitation $Z_{\mathrm{r}}\left(f_{\text {res }}\right)$ is purely ohmic. Consequently, the differential signal's amplitude can be written as (compare Fig. 6a)

$$
\begin{aligned}
\hat{V}_{\mathrm{d}}=\sqrt{2}\left|V_{\mathrm{d}}\left(f_{\text {res }}\right)\right| & =\frac{\hat{V}_{\text {ex }}\left|T\left(f_{\text {res }}\right)\right|\left|G\left(f_{\text {res }}\right)\right|}{Q\left(R_{\mathrm{r}}+R_{\text {mag }}\right)} \\
& =\frac{\hat{V}_{\text {ex }}\left|T\left(f_{\text {res }}\right)\right|}{R_{\mathrm{r}}+R_{\text {mag }}} .
\end{aligned}
$$

When neglecting the amplitude modulation and the static phase delays due to the sensor and the amplifiers, the associated time domain signal can be written as

$$
v_{\mathrm{d}}(t)=\hat{V}_{\mathrm{d}} \cos \left(2 \pi f_{\text {res }} t+S_{\mathrm{PM}} B_{\mathrm{x}}(t)+\varphi(t)\right)
$$

which contains the phase modulation with the phase sensitivity $S_{\mathrm{PM}}$ (Eq. (14)) and phase fluctuations $\varphi(t)$ due to the sensor and the electronics that are analyzed in more detail in the following section.

By means of a quadrature detector the phase demodulation is performed in the digital domain. For $f_{\text {ex }}=f_{\text {res }}$ the output signal is then equal to

$$
s_{\text {out }}(t)=S_{\mathrm{PM}} B_{\mathrm{x}}(t)+\varphi(t) .
$$

For all measurements in this paper, a high-resolution analogto-digital (A/D) and digital-to-analog (D/A) converter, respectively, of type Fireface UFX from RME running at a sampling rate of $32 \mathrm{kHz}$ has been used for digitizing $v_{\text {sensor }}(t)$ and $v_{\mathrm{n}}(t)$ and for generating the excitation signal $v_{\text {ex }}(t)$. The digital lowpass filters (LPF) in the quadrature detector are third-order Butterworth filters with $-3 \mathrm{~dB}$ cutoff frequencies of $3 \mathrm{kHz}$.

\section{Phase Noise Analysis}

\section{A. Thermal-mechanical and thermal-electrical noise}

As discussed in Sec. II-F, an electromechanical cantilever exhibits several loss mechanisms that, in the electrical equivalent circuit, are covered by two resistors with the resistances $R_{\mathrm{r}}+R_{\mathrm{mag}}$ and $R_{\mathrm{ME}}$. In previous studies [24], [25] it has already been shown that both the related thermal-mechanical noise of the resonant structure $E_{\mathrm{d} \text {,RrRmag as well as the }}$ thermal-electrical noise $E_{\mathrm{d}, \mathrm{RME}}$ of the dielectric material can accurately be predicted. Adjusting the previously published expressions to the readout structure as depicted in Fig. 5, the amount of the sensor's thermal voltage noise at the output of the subtractor can be calculated by

$$
\begin{aligned}
E_{\mathrm{d}, \operatorname{RrRmag}}(f) & =\left|\frac{T(f)}{Z_{\mathrm{r}}(f)}\right| \sqrt{4 k_{\mathrm{B}} T_{0}\left(R_{\mathrm{r}}+R_{\mathrm{mag}}\right)} \\
& =\frac{|T(f)||G(f)| \sqrt{4 k_{\mathrm{B}} T_{0}}}{Q \sqrt{R_{\mathrm{r}}+R_{\mathrm{mag}}}}
\end{aligned}
$$

and

$$
\begin{aligned}
E_{\mathrm{d}, \mathrm{RME}}(f) & =\frac{|T(f)|}{R_{\mathrm{ME}}(f)} \sqrt{4 k_{\mathrm{B}} T_{0} R_{\mathrm{ME}}(f)} \\
& =\frac{|T(f)| \sqrt{4 k_{\mathrm{B}} T_{0}}}{\sqrt{R_{\mathrm{ME}}(f)}}
\end{aligned}
$$

where $k_{\mathrm{B}} \approx 1.381 \cdot 10^{-23} \mathrm{~J} / \mathrm{K}$ is the Boltzmann constant and $T_{0}=290 \mathrm{~K}$ the room temperature. A noise measurement without any external excitation of the sensor $\left(\hat{V}_{\mathrm{ex}}=0\right)$ and in comparison to the theoretical expectations is depicted in Fig. 7. Due to the sensor's resonant behavior the thermal-mechanical noise $E_{\mathrm{d}, \mathrm{RrRmag}}$ is weighted by $G(f)$ and perfectly agrees 


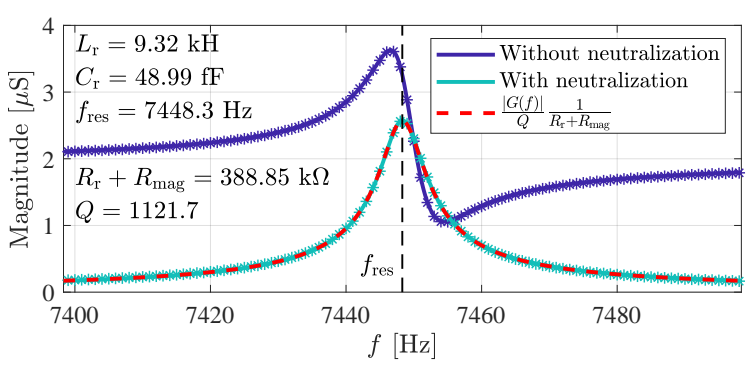

(a) Magnitude of the sensor's admittance

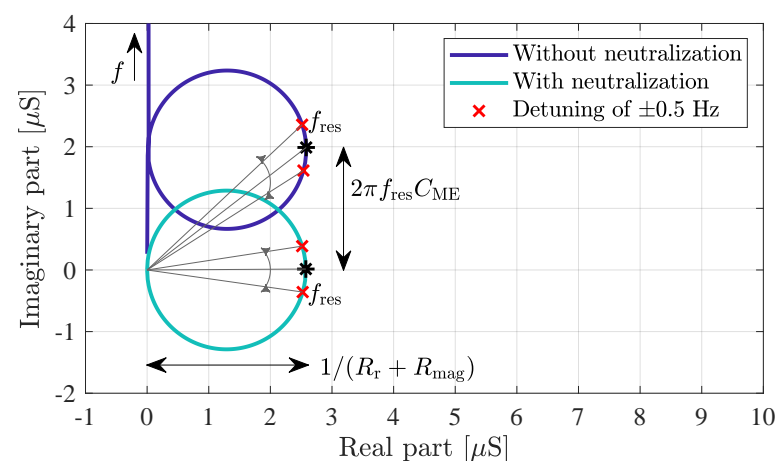

(c) Nyquist plot of the sensor's admittance

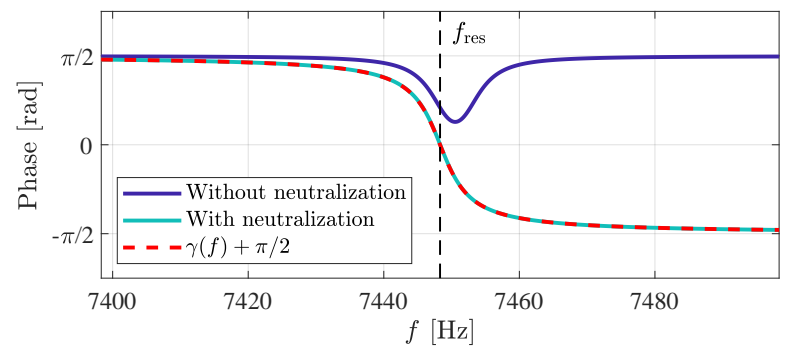

(b) Phase of the sensor's admittance

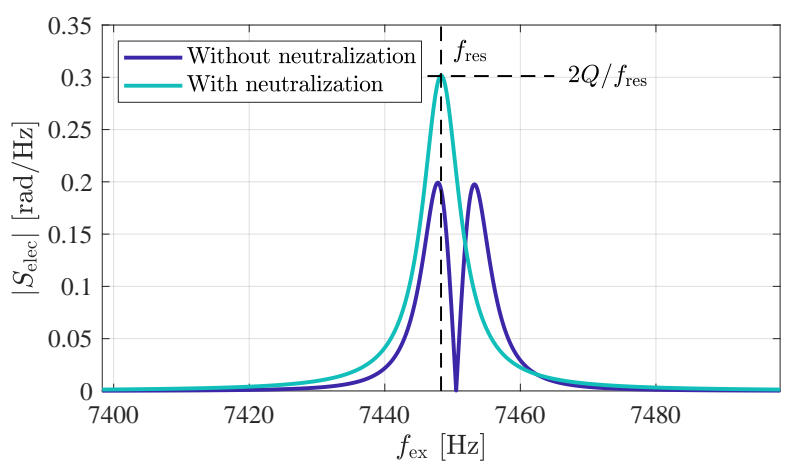

(d) Electrical sensitivity

Fig. 6: Measured (stars) and calculated (solid lines) trajectories of magnitude (a) and phase (b) of the sensor's admittance $Y_{\text {sensor. }}$ Due to the parasitic influence of the sensor's static capacitance $C_{\mathrm{ME}}$ both line shapes are distorted, i.e. the locus curve (c) is shifted to higher imaginary parts. Neutralizing this effect not only leads to symmetric line shapes but also to an increase in the electrical sensitivity (d). The measurements have been conducted in magnetic saturation and for $\hat{V}_{\text {ex }}=1 \mathrm{mV}$.

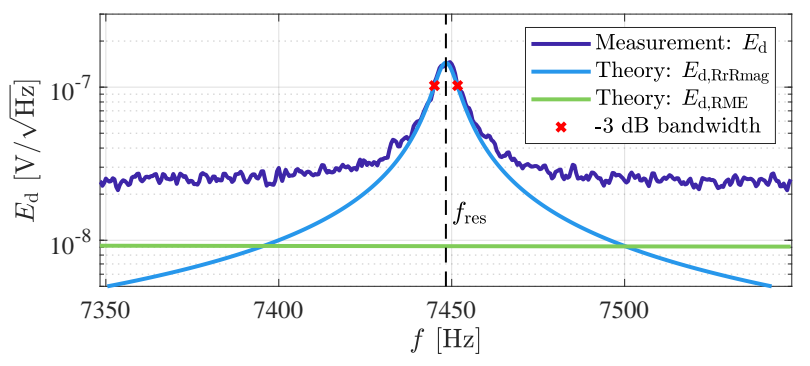

Fig. 7: Measured noise at the output of the subtractor in comparison to the theoretical expectations according to Eq. (27) and (29). In the vicinity of the resonance frequency the overall noise is dominated by the sensor-intrinsic thermal-mechanical noise $E_{\mathrm{d}, \mathrm{RrRmag}}$. Far beyond the sensor's $-3 \mathrm{~dB}$ bandwidth the measured noise is approximately frequency independent but higher than predicted $\left(E_{\mathrm{d}, \mathrm{RME}}\right)$ because of further contributions of the system electronics. The measurement has been conducted in magnetic saturation and for $\hat{V}_{\mathrm{ex}}=0$.

with the measurement in the vicinity of the resonance frequency. Far beyond the sensor's $-3 \mathrm{~dB}$ bandwidth the measured noise is approximately frequency independent but higher than predicted $\left(E_{\mathrm{d}, \mathrm{RME}}\right)$ because of further contributions due to the system electronics, i.e. that of the two charge amplifiers and the D/A and A/D converters, respectively.

\section{B. Relation between voltage noise density and phase noise}

Various and statistically independent voltage noise densities due to the sensor and the system electronics $\left(E_{\mathrm{d}, \text { system }}\right)$ add up at the output of the subtractor

$$
E_{\mathrm{d}}^{2}=E_{\mathrm{d}, \mathrm{RrRmag}}^{2}+E_{\mathrm{d}, \mathrm{RME}}^{2}+E_{\mathrm{d}, \text { system }}^{2}
$$

To determine their relation to the power spectral density $S_{\varphi}(f)$ of the random phase fluctuations $\varphi(t)$, Eq. (24) is written as

$$
v_{\mathrm{d}}(t)=\hat{V}_{\mathrm{d}} \cos \left(2 \pi f_{\mathrm{res}} t+\hat{\varphi}\left(f_{\mathrm{x}}\right) \cos \left(2 \pi f_{\mathrm{x}} t\right)\right)
$$

in which one noise component with the modulation index $\hat{\varphi}$ at $f_{\mathrm{x}}$ represents other spectral components that can be taken into account by linear superposition. In addition, for this noise consideration the measurement signal $B_{\mathrm{x}}(t)$ is assumed to be zero. Based on basic trigonometric identities Eq. (31) can be rearranged into

$$
\begin{gathered}
v_{\mathrm{d}}(t)=\hat{V}_{\mathrm{d}}\left[\cos \left(2 \pi f_{\text {res }} t\right)-\frac{\hat{\varphi}\left(f_{\mathrm{x}}\right)}{2}\left[\sin \left(2 \pi\left(f_{\text {res }}-f_{\mathrm{x}}\right) t\right)\right.\right. \\
\left.\left.+\sin \left(2 \pi\left(f_{\text {res }}+f_{\mathrm{x}}\right) t\right)\right]\right]
\end{gathered}
$$

revealing the typical structure of a narrow band small signal phase modulated signal with a carrier at $f_{\text {res }}$ and two symmet- 
rical sidebands at $f_{\text {res }} \pm f_{\mathrm{x}}$. Following the concept of noise sidebands [63, pp. 243] the carrier-to-noise sideband ratio

$$
\frac{\hat{V}_{\mathrm{d}}}{\hat{V}_{\mathrm{d}} \frac{\hat{\varphi}\left(f_{\mathrm{x}}\right)}{2}}=\frac{2}{\hat{\varphi}\left(f_{\mathrm{x}}\right)}=\frac{\hat{V}_{\mathrm{d}}}{E_{\mathrm{d}}\left(f_{\mathrm{res}} \pm f_{\mathrm{x}}\right) \sqrt{2} \sqrt{\Delta f}}
$$

is equal to the carrier-to-voltage noise ratio if a symmetrical noise distribution $\left(E_{\mathrm{d}}\left(f_{\mathrm{res}}-f_{\mathrm{x}}\right)=E_{\mathrm{d}}\left(f_{\text {res }}+f_{\mathrm{x}}\right)\right)$ around the resonance frequency is assumed. The additional term $\sqrt{\Delta f}$ transforms the voltage noise density into an effective voltage noise in the bandwidth $\Delta f$. From Eq. (33) the phase modulation index

$$
\hat{\varphi}\left(f_{\mathrm{x}}\right)=\frac{2 E_{\mathrm{d}}\left(f_{\mathrm{res}} \pm f_{\mathrm{x}}\right) \sqrt{2} \sqrt{\Delta f}}{\hat{V}_{\mathrm{d}}}
$$

can be deduced which directly yields the power spectral density

$$
S_{\varphi}\left(f_{\mathrm{x}}\right)=\left(\frac{2 E_{\mathrm{d}}\left(f_{\mathrm{res}} \pm f_{\mathrm{x}}\right)}{\hat{V}_{\mathrm{d}}}\right)^{2}
$$

of the random phase fluctuations $\varphi(t)$ in units of $\operatorname{rad}^{2} / \mathrm{Hz}$. For voltage noise distributed asymmetrically around the resonance frequency $\left(E_{\mathrm{d}}\left(f_{\text {res }}-f_{\mathrm{x}}\right) \neq E_{\mathrm{d}}\left(f_{\text {res }}+f_{\mathrm{x}}\right)\right)$ the more generally valid power spectral density is given by

$$
S_{\varphi}\left(f_{\mathrm{x}}\right)=\left(\frac{E_{\mathrm{d}}\left(f_{\mathrm{res}}-f_{\mathrm{x}}\right)+E_{\mathrm{d}}\left(f_{\mathrm{res}}+f_{\mathrm{x}}\right)}{\hat{V}_{\mathrm{d}}}\right)^{2} .
$$

However, for the frequency range in the vicinity of $f_{\text {res }}$

$$
\left|T\left(f_{\text {res }}\right)\right| \approx \frac{1}{2}\left(\left|T\left(f_{\text {res }}-f_{\mathrm{x}}\right)\right|+\left|T\left(f_{\text {res }}+f_{\mathrm{x}}\right)\right|\right)
$$

and

$$
R_{\mathrm{ME}}\left(f_{\mathrm{res}}\right) \approx \frac{1}{2}\left(R_{\mathrm{ME}}\left(f_{\mathrm{res}}-f_{\mathrm{x}}\right)+R_{\mathrm{ME}}\left(f_{\mathrm{res}}+f_{\mathrm{x}}\right)\right)
$$

are generally good approximations. Because of the neutralization $E_{\mathrm{d}, \mathrm{RrRmag}}(f)$ is also symmetric around $f_{\text {res }}$, thus leading to expressions for the power spectral densities of random phase fluctuations due to thermal-mechanical noise

$$
\begin{gathered}
S_{\varphi, \text { RrRmag }}\left(f_{\mathrm{x}}\right)=\left(\frac{2\left|T\left(f_{\mathrm{res}}\right) S_{\mathrm{dyn}}\left(f_{\mathrm{x}}\right)\right| \sqrt{4 k_{\mathrm{B}} T_{0}}}{\hat{V}_{\mathrm{d}} \sqrt{R_{\mathrm{r}}+R_{\mathrm{mag}}}}\right)^{2} \\
\stackrel{\text { Eq. (23) }}{=}\left(\frac{2\left|S_{\mathrm{dyn}}\left(f_{\mathrm{x}}\right)\right| \sqrt{4 k_{\mathrm{B}} T_{0}\left(R_{\mathrm{r}}+R_{\mathrm{mag}}\right)}}{\hat{V}_{\mathrm{ex}}}\right)^{2}
\end{gathered}
$$

and due to thermal-electrical noise of the loss in the dielectric material

$$
\begin{aligned}
& S_{\varphi, \mathrm{RME}}=\left(\frac{2\left|T\left(f_{\mathrm{res}}\right)\right| \sqrt{4 k_{\mathrm{B}} T_{0}}}{\hat{V}_{\mathrm{d}} \sqrt{R_{\mathrm{ME}}\left(f_{\mathrm{res}}\right)}}\right)^{2} \\
& \stackrel{\text { Eq. (23) }}{=}\left(\frac{2 \sqrt{4 k_{\mathrm{B}} T_{0}}\left(R_{\mathrm{r}}+R_{\mathrm{mag}}\right)}{\hat{V}_{\mathrm{ex}} \sqrt{R_{\mathrm{ME}}\left(f_{\mathrm{res}}\right)}}\right)^{2} .
\end{aligned}
$$

Obviously, the thermal-electrical noise $E_{\mathrm{d}, \mathrm{RME}}$ leads to additive white phase noise that decreases with the excitation amplitude since $\hat{V}_{\mathrm{d}} \propto \hat{V}_{\text {ex }}$. The phase noise, which is caused by the thermal-mechanical noise $E_{\mathrm{d}, \mathrm{RrRmag}}$, also decreases with $\hat{V}_{\text {ex }}$. However, due to the influence of the resonator this additive phase noise decreases with $20 \mathrm{~dB} /$ decade for frequencies $f_{\mathrm{x}}$ above the cutoff frequency $f_{\mathrm{c}}=f_{\mathrm{res}} /(2 Q)$. In contrast to this, thermal-mechanical noise also leads to white phase noise for frequencies well below the cutoff frequency $\left(f_{\mathrm{x}} \ll f_{\mathrm{c}}\right)$.

\section{Phase noise measurements}

With the system described above (Fig. 5), several series of noise measurements were performed to analyze the sensor's phase noise behavior. Without any additional magnetic measurement signal $\left(B_{\mathrm{x}}(t)=0\right)$, the system's output signal $s_{\text {out }}(t)$ is then equal to the random phase fluctuations $\varphi(t)$ which were transformed to the frequency domain based on Welch's method [64], thus leading to the power spectral densities of random phase fluctuations $S_{\varphi}$. In the following, $S_{\varphi}\left(f_{\mathrm{x}}\right)$ is given as a function of the frequency $f_{\mathrm{x}}$ to clarify that this phase noise is effective in the same frequency range as the measurement signal. Formally it would be just as correct to use $f$ here.

For all measurements the sensor was placed inside an ultrahigh magnetic field shielding mu-metal cylinder (Aaronia AG ZG1) which, in turn, is placed inside a vibrationally decoupled box with lined absorbers against airborne sound. In addition, the whole box is coated with a copper fleece, which shields electrical fields. Furthermore, the sensor is surrounded by two solenoids inside the mu-metal that are used for generating both the magnetic bias flux density $B_{\text {bias }}$ as well as the magnetic measurement signal $B_{\mathrm{x}}(t)$. The latter is generated with a commercially available current source (Keithley 6221). However, no commercially available power source was suitable for generating comparatively large bias fields in the millitesla range while keeping the resulting low-frequency noise well below $100 \mathrm{pT} / \sqrt{\mathrm{Hz}}$. Therefore, an in-house built setup based on several batteries (capacity $>100 \mathrm{Ah}$ ) and a stepper motor controlled potentiometer in series to the coil was used.

In a first series of noise measurements the sensor was magnetically saturated by means of a strong permanent magnet generating a magnetic bias flux density of $B_{\text {bias }} \approx 20 \mathrm{mT}$ which is distinctly higher than the sensor's saturation flux density $<5 \mathrm{mT}$ (compare e.g. Fig. 2a). At the same time the amplitude $\hat{V}_{\mathrm{ex}}$ of the electrical excitation signal $V_{\mathrm{ex}}(t)$ was increased incrementally. For several values of $\hat{V}_{\text {ex }}$, the measured phase noise is depicted in Fig. 8a together with the phase noise contributions due to thermal-mechanical noise $S_{\varphi, \text { RrRmag }}$ (Eq. (40), dashed lines) and due to thermalelectrical noise of the dielectric material $S_{\varphi, \mathrm{RME}}$ (Eq. (42), dotted lines). As expected, the measured noise spectra decrease with increasing excitation amplitudes, thus confirming the additive character of both thermal noise contributions. In addition, values of measured phase noise and calculated phase noise $S_{\varphi, \text { RrRmag }}$ (dashed lines) agree well for the low frequency range in the range of the sensor's passband, indicated by the respective cutoff frequency (black crosses). Due to the impact of the mechanical resonator, the phase noise decreases with higher frequencies proportional to $f_{\mathrm{x}}^{-2}$ until the white phase noise floor beyond the sensor's passband is reached. According to previous calculations, the crossover frequency between frequency-dependent and white 


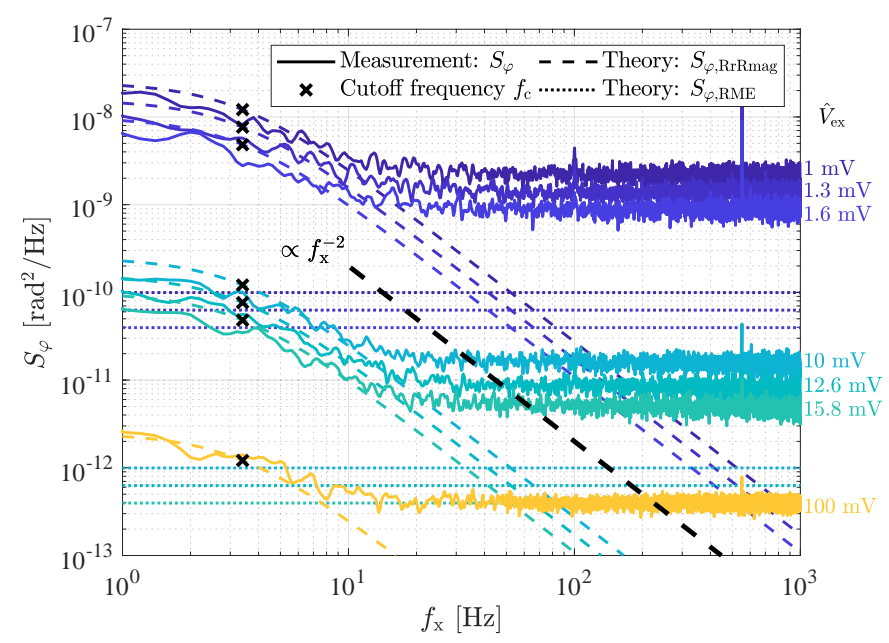

(a) Sensor in magnetic saturation

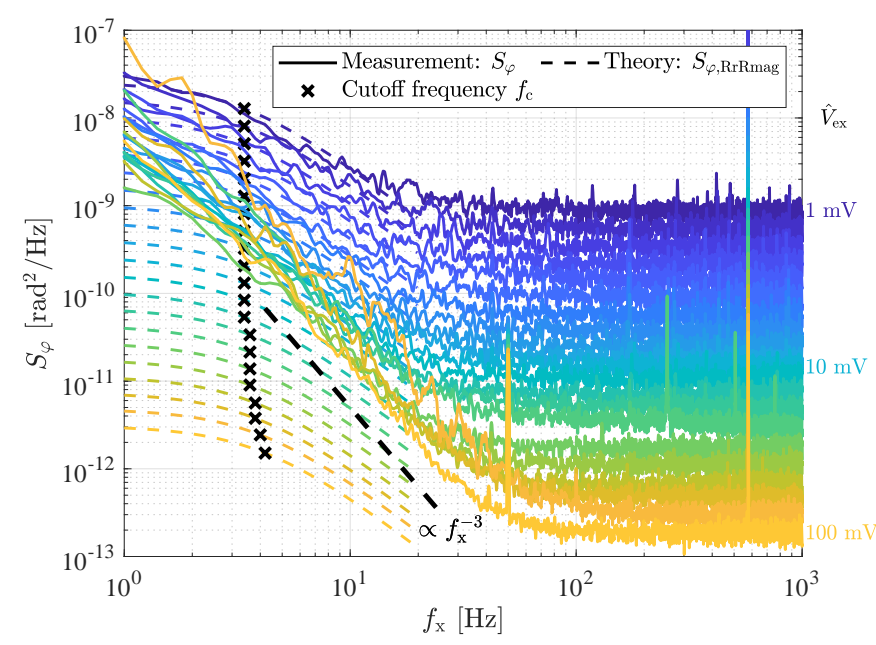

(b) Sensor in magnetic operating point $B_{\text {bias }}=0.65 \mathrm{mT}$

Fig. 8: Power spectral densities of the measured random phase fluctuations $\varphi(t)$ at the sensor system's output for the sensor in magnetic saturation (a) and for the sensor in its magnetic operating point (b) for various amplitudes of the excitation signal. For the magnetically saturated sensor, the additive phase noise due to thermal-mechanical (Eq. (40), dashed lines) and due to thermal-electrical noise (Eq. (42), dotted lines) decreases with the excitation amplitude $\hat{V}_{\text {ex }}$. For the sensor in its magnetic operating point low-frequency parametric phase noise occurs whose underlying physical noise process must exhibit a $f_{\mathrm{x}}^{-1}$ characteristic because an amount of $f_{\mathrm{x}}^{-2}$ is attributed to the influence of the resonator. The slightly different levels of white phase noise floors between the measurements in (a) and (b) are probably due to different states of charge of the battery supplying the amplifier, thus leading to different drain currents in the amplifier's discrete JFET front-end [25].

phase noise due to thermal-electrical noise of the dielectric material $\left(S_{\varphi, \mathrm{RrRmag}}=S_{\varphi, \mathrm{RME}}\right)$ is about $50 \mathrm{~Hz}$. However, as already discussed in the context of Fig. 7, the overall noise floor outside the sensor's passband is dominated by noise contributions of the system electronics, thus leading to a crossover frequency at about $10 \mathrm{~Hz}$ for the system under investigation.

The same series of measurements was repeated for the sensor not being saturated anymore but brought into its magnetic operating point of $B_{\text {bias }}=0.65 \mathrm{mT}$ after saturating the sensor in negative direction and stepwise incrementing $B_{\text {bias. }}$. The measured phase noise acquired in this way is shown in Fig. 8b, again in comparison to the theoretical expectations (dashed lines) according to Eq. (40) (phase noise due to thermalmechanical noise $S_{\varphi, \text { RrRmag }}$ ). The measured phase noise at low excitation amplitudes of about $1 \mathrm{mV}$ still corresponds to the contribution of the thermal-mechanical noise. However, for increasing amplitudes $\hat{V}_{\text {ex }}$ the measured phase noise in the sensor's passband no longer decreases significantly as for the magnetically saturated case (Fig. 8a). Thus, the noise contribution of the magnetic material leads to so-called parametric noise which is independent from the amplitude of the carrier signal [65, p. 36], at least if the noise process itself or material properties do not depend on the amplitude. Such a behavior is well-known from $1 / f$ flicker phase noise, e.g. of amplifiers [66]. And indeed, considering the slope of $f_{\mathrm{x}}^{-3}$ with which the measured phase noise decreases, the underlying physical noise process must exhibit a $f_{\mathrm{x}}^{-1}$ characteristic because an amount of $f_{\mathrm{x}}^{-2}$ is attributed to the influence of the resonator.
To further verify the relationship between the sensor's magnetic state and the magnetically induced phase noise, measurements as a function of the magnetic bias flux density $B_{\text {bias }}$ from negative to positive saturation were conducted (inverse measurement gives results mirrored on the axis of ordinates). As depicted by the corresponding power spectral densities of the random phase fluctuations in Fig. 9a, the $f_{\mathrm{x}}^{-1}$ flicker phase noise and the $f_{\mathrm{x}}^{-3}$ phase noise at the sensor system's output, respectively, clearly depend on the sensor's magnetic state. In fact, as shown by the measured phase noise at a frequency of $1 \mathrm{~Hz}$ in Fig. 9b, the induced phase noise is unambiguously related to the magnetic losses,

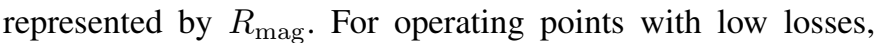
i.e. at which the magnetic sensitivity is low, e.g. near saturation and for $B_{\text {bias }}=0$, also the phase noise adopts lower values. In contrast, the phase noise is particularly high when the losses or the magnetic sensitivity is high (dashed lines at $B_{\text {bias }}= \pm 0.65 \mathrm{mT}$ ). In investigations on magnetoresistive sensors, an identical behavior could be observed in the past [67], [68]. These sensors also show largest noise for operating points of maximum sensitivity which was attributed to random fluctuations of the magnetization due to magnetic domain wall movements and rotations [68], [69].

\section{Magnetically induced flicker phase noise}

Due to the significant relation to the magnetic losses it is obvious to describe the magnetically induced phase noise using the fluctuation-dissipation theorem. Based on that theo- 


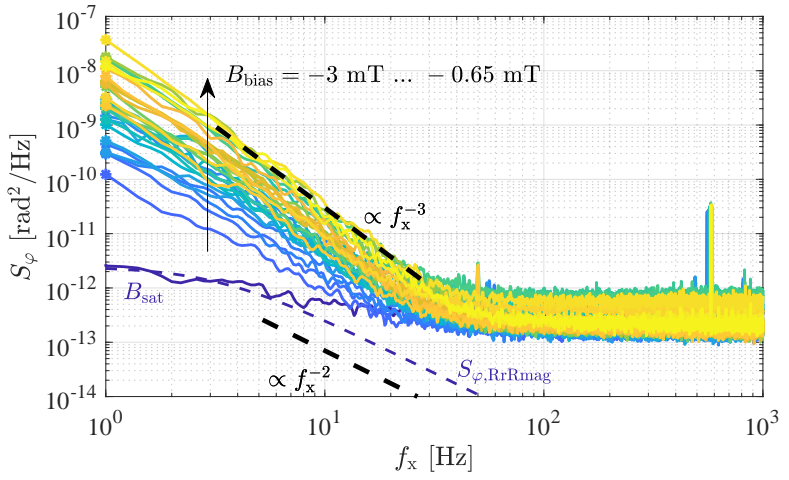

(a) Phase noise for various magnetic bias flux densities in the frequency domain

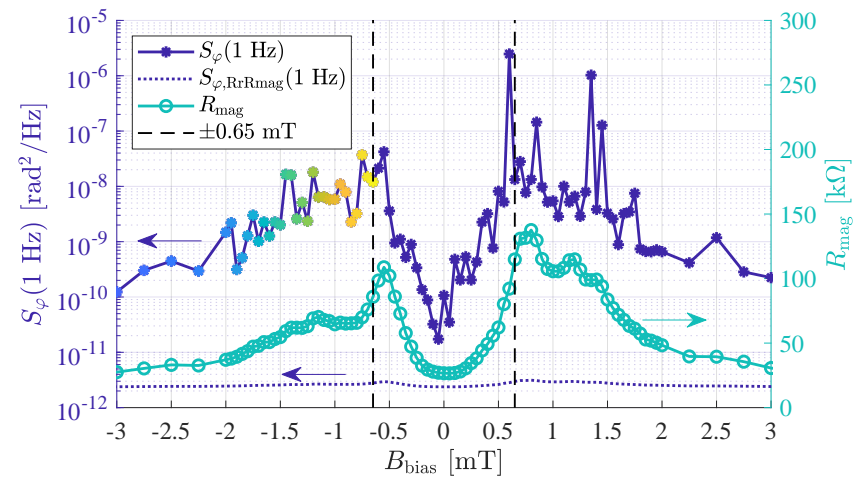

(b) Phase noise at a frequency of $1 \mathrm{~Hz}$ and magnetic losses as a function of the magnetic bias flux density

Fig. 9: Power spectral densities of the measured random phase fluctuations $\varphi(t)$ at the sensor system's output for a constant excitation amplitude of $\hat{V}_{\text {ex }}=100 \mathrm{mV}$ as a function of the magnetic bias flux density $B_{\text {bias }}$ measured increasingly from negative to positive magnetic saturation. The magnetically induced phase noise significantly changes with $B_{\text {bias }}$ while the slope stays constant as far as the sensor is not in magnetic saturation (a). Obviously, the measured phase noise is directly linked to the magnetic losses, represented by $R_{\text {mag }}$, and for this excitation amplitude always higher than the phase noise due to the sensor's thermal-mechanical noise $S_{\varphi, \text { RrRmag }}(\mathrm{b})$.

rem, the power spectral density of random fluctuations of the magnetization $M$

$$
S_{\mathrm{M}}\left(f_{\mathrm{x}}\right)=\frac{4 k_{\mathrm{B}} T_{0}}{2 \pi f_{\mathrm{x}} V_{\mathrm{mag}}} \frac{\mu_{\mathrm{r}, \mathrm{eff}}^{\prime \prime}}{\mu_{0}}
$$

with the physical dimension $(\mathrm{A} / \mathrm{m})^{2} / \mathrm{Hz}$ can be derived [70], [71] which can be referred to as flicker magnetization noise since the power density decreases with $1 / f_{\mathrm{x}}$. This expression is typically given as a function of the imaginary part $\mu_{\mathrm{r}}^{\prime \prime}$ of the magnetic material's complex permeability $\mu_{\mathrm{r}}=\mu_{\mathrm{r}}^{\prime}-j \mu_{\mathrm{r}}^{\prime \prime}$. In general, however, $\mu_{\mathrm{r}}^{\prime \prime}$ is also used to account for other losses, in particular eddy current losses, which in turn do not correspond with flicker noise but with frequency-independent white noise [72]. Therefore, in this paper, an effective complex permeability $\mu_{\mathrm{r}, \mathrm{eff}}=\mu_{\mathrm{r}}^{\prime}-j \mu_{\mathrm{r} \text {, eff }}^{\prime \prime}$ is used to cover only for magnetic hysteresis losses corresponding with $1 / f$ flicker noise. Furthermore, $\mu_{0}=4 \pi \cdot 10^{-7} \mathrm{Vs} /(\mathrm{Am})$ and $V_{\text {mag }}$ denote the vacuum permeability and the volume of the magnetic material, respectively. Thus, fluctuations of the magnetization can be decreased by larger magnetic volumes, at least if the magnetic losses $\mu_{\mathrm{r}, \mathrm{eff}}^{\prime \prime}$ do not rise proportionally with $V_{\mathrm{mag}}$. However, literature shows that volume and losses are generally not independent of each other [73].

The expression for changes of the resonant sensor's phase response $\gamma(f)$ (Eq. 8) at the resonance frequency $f_{\text {res }}$ due to changes of the magnetization $M$

$$
\frac{\partial \gamma\left(f_{\text {res }}\right)}{\partial M}=\left.\frac{\partial \gamma(f)}{\partial f}\right|_{f=f_{\text {res }}} \frac{\partial f_{\text {res }}}{\partial M}
$$

can be factorized into two terms. The first term describes the changes of the sensor's phase response at $f_{\text {res }}$ due to a detuning of the resonator. As discussed above, this term is equal to the electrical sensitivity $S_{\text {elec }}$ (Eq. (9)). The second term covers the detuning of the resonator due to changes of the magnetization. With the magnetic susceptibility $\chi=\partial M / \partial H=\mu_{\mathrm{r}}^{\prime}-1$, with $B=\mu_{0} H$, and with $S_{\text {mag }}=\partial f_{\text {res }} / \partial B$ (Eq. (5)) the second term

$$
\begin{aligned}
\frac{\partial f_{\text {res }}}{\partial M} & =\frac{\partial f_{\text {res }}}{\partial H} \frac{\partial H}{\partial M}=\frac{\partial f_{\text {res }}}{\partial H} \frac{1}{\chi}=\frac{\partial f_{\text {res }}}{\mu_{0} \partial H} \frac{\mu_{0}}{\chi} \\
& =S_{\text {mag }} \frac{\mu_{0}}{\chi} \approx S_{\operatorname{mag}} \frac{\mu_{0}}{\mu_{\mathrm{r}}^{\prime}}
\end{aligned}
$$

can be expressed as a function of the magnetic sensitivity $S_{\text {mag }}$ and the real part $\mu_{\mathrm{r}}^{\prime}$ of the effective permeability. The approximation is generally valid for commonly utilized magnetic materials with high permeabilities $\left(\mu_{\mathrm{r}}^{\prime} \gg 1\right)$.

Using these relations, the power spectral density of random phase fluctuations due to random fluctuations of the magnetization yields

$$
\begin{aligned}
& S_{\varphi, \mathrm{M}}\left(f_{\mathrm{x}}\right)=S_{\mathrm{M}}\left(f_{\mathrm{x}}\right)\left|\frac{\partial \gamma\left(f_{\mathrm{res}}\right)}{\partial M}\right|^{2}\left|S_{\mathrm{dyn}}\left(f_{\mathrm{x}}\right)\right|^{2} \\
& =S_{\mathrm{M}}\left(f_{\mathrm{x}}\right)\left|S_{\text {elec }}\right|^{2}\left|S_{\text {mag }} \frac{\mu_{0}}{\mu_{\mathrm{r}}^{\prime}}\right|^{2}\left|S_{\mathrm{dyn}}\left(f_{\mathrm{x}}\right)\right|^{2}
\end{aligned}
$$

in which the dynamic sensitivity $S_{\text {dyn }}$ (Eq. (13)) accounts for the additional decrease in phase noise with increasing frequency due to the resonator. With Eq. (43) and the sensor's overall phase sensitivity $S_{\mathrm{PM}}$ (Eq. (14)) the expression further simplifies to

$$
S_{\varphi, \mathrm{M}}\left(f_{\mathrm{x}}\right)=\frac{4 k_{\mathrm{B}} T_{0}}{2 \pi f_{\mathrm{x}} V_{\mathrm{mag}}}\left|S_{\mathrm{PM}}\left(f_{\mathrm{x}}\right)\right|^{2} \frac{\mu_{0} \mu_{\mathrm{r}, \mathrm{eff}}^{\prime \prime}}{\left(\mu_{\mathrm{r}}^{\prime}\right)^{2}},
$$

clarifying that the magnetically induced phase noise is proportional to the sensor's sensitivity.

\section{E. Limit of detection}

The limit of detection (LOD) of a magnetic field sensor system denotes the frequency-dependent noise floor, i.e. an 


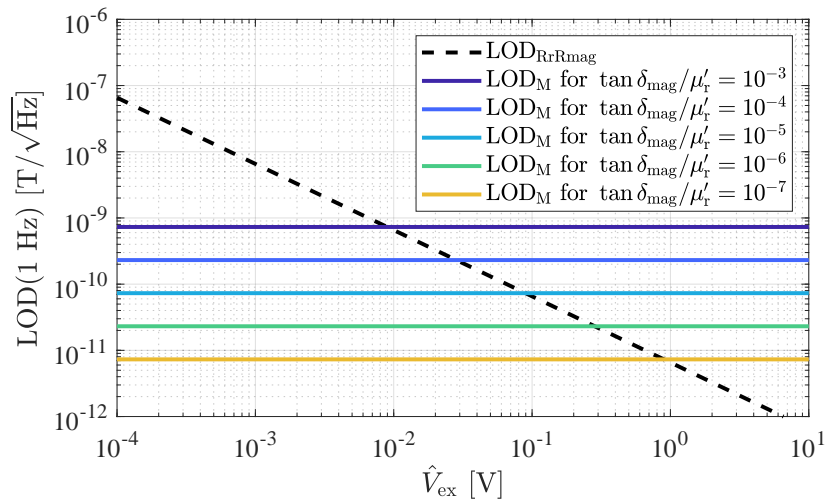

Fig. 10: Calculated limit of detection (LOD) at a frequency of $1 \mathrm{~Hz}$ and for typical sensor parameters $\left(f_{\text {res }}=7450 \mathrm{~Hz}\right.$, $R_{\mathrm{r}}+R_{\mathrm{mag}}=388.85 \mathrm{k} \Omega, Q=1121.7, \quad S_{\mathrm{mag}}=80 \mathrm{~Hz} / \mathrm{mT}$, $\left.V_{\text {mag }}=6 \cdot 10^{-12} \mathrm{~m}^{3}\right)$ at room temperature $\left(T_{0}=290 \mathrm{~K}\right)$. The fundamental LOD is limited by additive thermal-mechanical

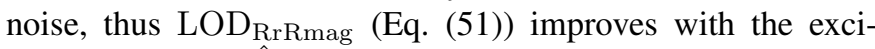
tation amplitude $\hat{V}_{\text {ex }}$. Parametric magnetically induced phase noise $S_{\varphi, \mathrm{M}}$ (Eq. (49)) limits the LOD of real sensors. However, LOD $_{M}$ (Eq. (52) and (54)) can be improved by decreasing the relative magnetic loss factor $\tan \delta_{\mathrm{mag}} / \mu_{\mathrm{r}}^{\prime}$.

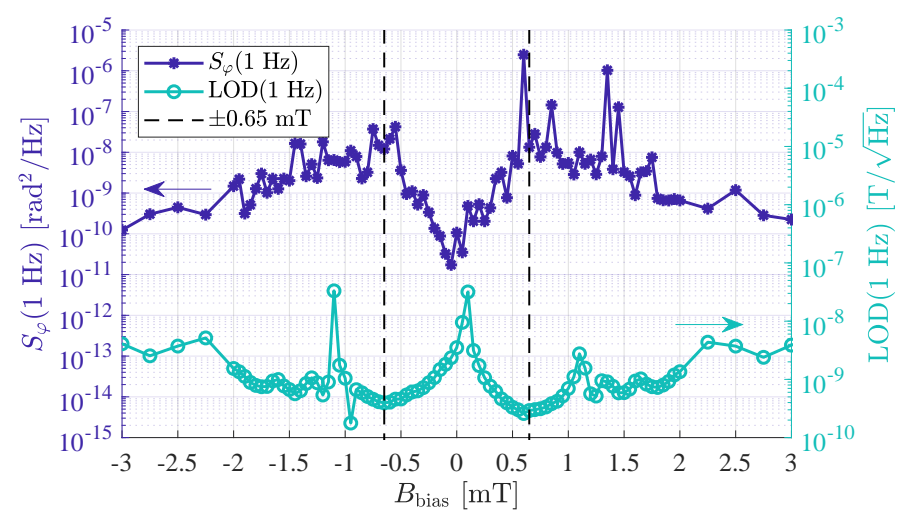

Fig. 11: Measured phase noise at a frequency of $1 \mathrm{~Hz}$ (same data as in Fig. 9b) and measured limit of detection (LOD), also at a frequency of $1 \mathrm{~Hz}$ for a constant excitation amplitude of $\hat{V}_{\text {ex }}=100 \mathrm{mV}$ as a function of $B_{\text {bias }}$ measured increasingly from negative to positive magnetic saturation. Best values as low as $\operatorname{LOD}(1 \mathrm{~Hz})=292 \mathrm{pT} / \sqrt{\mathrm{Hz}}$ are obtained in the sensor's magnetic operating point at $B_{\text {bias }}=0.65 \mathrm{mT}$.

amplitude spectral density, in units of $\mathrm{T} / \sqrt{\mathrm{Hz}}$ [46]. Thus, the LOD is given by the ratio of the amplitude spectral density of random phase fluctuations and the phase sensitivity.

Considering only the phase noise $S_{\varphi, \text { RrRmag }}$ (Eq. (40)) due to the resonator's thermal-mechanical noise, the fundamental LOD is given by

$$
\begin{aligned}
\operatorname{LOD}_{\operatorname{RrRmag}}\left(f_{\mathrm{x}}\right) & =\frac{\sqrt{S_{\varphi, \operatorname{RrRmag}}\left(f_{\mathrm{x}}\right)}}{\left|S_{\mathrm{PM}}\left(f_{\mathrm{x}}\right)\right|} \\
& =\frac{f_{\text {res }} \sqrt{4 k_{\mathrm{B}} T_{0}\left(R_{\mathrm{r}}+R_{\text {mag }}\right)}}{\hat{V}_{\mathrm{ex}} Q\left|S_{\mathrm{mag}}\right|}
\end{aligned}
$$

which is frequency-independent, deteriorates with the losses, and improves with the magnetic sensitivity. In particular, this fundamental LOD could be improved simply by increasing the excitation amplitude because the phase noise due to thermal-mechanical noise is additive (Fig. 8a). For the sensor under investigation, with typical values (compare Fig. 6a) of $f_{\text {res }}=7450 \mathrm{~Hz}, R_{\mathrm{r}}+R_{\text {mag }}=388.85 \mathrm{k} \Omega, Q=1121.7$, and $S_{\mathrm{mag}}=80 \mathrm{~Hz} / \mathrm{mT}$ the frequency-independent LOD would result, e.g. in a value as low as $6.5 \mathrm{pT} / \sqrt{\mathrm{Hz}}$ for an electrical driving amplitude of $\hat{V}_{\text {ex }}=1 \mathrm{~V}$ (dashed line in Fig. 10).

However, because the sensor system's overall noise floor is dominated by magnetically induced phase noise (as shown in Fig. 9b this noise is usually distinctly higher than phase noise due to the resonator's thermal-mechanical noise when the magnetic material is not saturated) it is more convenient to consider $S_{\varphi, \mathrm{M}}\left(f_{\mathrm{x}}\right)$ (Eq. (49)) for the determination of the detectivity

$$
\operatorname{LOD}_{\mathrm{M}}\left(f_{\mathrm{x}}\right)=\frac{\sqrt{S_{\varphi, \mathrm{M}}\left(f_{\mathrm{x}}\right)}}{S_{\mathrm{PM}}\left(f_{\mathrm{x}}\right)}=\sqrt{\frac{4 k_{\mathrm{B}} T_{0}}{2 \pi f_{\mathrm{x}} V_{\mathrm{mag}}} \frac{\mu_{0} \mu_{\mathrm{r}, \mathrm{eff}}^{\prime \prime}}{\left(\mu_{\mathrm{r}}^{\prime}\right)^{2}}}
$$

which improves with $1 / \sqrt{f_{\mathrm{x}}}$. Remarkably, in that case, the limit of detection does not depend on the sensor's sensitivity at all but is solely determined by the volume and the magnetic properties $\mu_{\mathrm{r}}^{\prime}$ and $\mu_{\mathrm{r}, \text { eff }}^{\prime \prime}$ of the magnetostrictive film which, in turn, depend on the sensor's operating point in terms of bias field, excitation frequency, and excitation power. A basically identical result was reported e.g. for giant magnetoimpedance sensors, for which the fundamental detectivity is also independent of the sensitivity [74]. A recently published article [38] about magnetic domain activities confirms the relation between magnetic losses and magnetic noise in periodically driven magnetoelectric cantilevers. The authors also come to the conclusion that controlling the magnetic domain behavior is the key to optimum sensor performance.

For the sensor under investigation magnetically coated with a volume of $V_{\mathrm{mag}}=3 \mathrm{~mm} \cdot 1 \mathrm{~mm} \cdot 2 \mu \mathrm{m}=6 \cdot 10^{-12} \mathrm{~m}^{3}$, the expression for the LOD can be further simplified to

$$
\begin{aligned}
\operatorname{LOD}_{\mathrm{M}}\left(f_{\mathrm{x}}\right) & =\frac{23.1 \mathrm{nT}}{\sqrt{f_{\mathrm{x}}}} \frac{\sqrt{\mu_{\mathrm{r}, \mathrm{eff}}^{\prime \prime}}}{\mu_{\mathrm{r}}^{\prime}} \\
& =\frac{23.1 \mathrm{nT}}{\sqrt{f_{\mathrm{x}}}} \sqrt{\frac{\tan \delta_{\mathrm{mag}}}{\mu_{\mathrm{r}}^{\prime}}},
\end{aligned}
$$

clarifying the exclusive dependence on the magnetostrictive film's magnetic properties. The best value for the detectivity of $\operatorname{LOD}(1 \mathrm{~Hz})=292 \mathrm{pT} / \sqrt{\mathrm{Hz}}$ is measured around a bias flux density of $B_{\text {bias }}=+0.65 \mathrm{mT}$ (Fig. 11) despite the higher losses compared to the operating point around $B_{\text {bias }}=-0.65 \mathrm{mT}$ (Fig. 9b) for which a value of $\operatorname{LOD}(1 \mathrm{~Hz})=394 \mathrm{pT} / \sqrt{\mathrm{Hz}}$ is achieved. The reason is the higher magnetic sensitivity in this measurement of $S_{\text {mag }}=61.3 \mathrm{~Hz} / \mathrm{mT}$ at $B_{\text {bias }}=+0.65 \mathrm{mT}$ compared to a value of $S_{\mathrm{mag}}=42.5 \mathrm{~Hz} / \mathrm{mT}$ at $B_{\text {bias }}=-0.65 \mathrm{mT}$. Thus, an optimum LOD is achieved at an operating point at which the sensitivity-to-loss ratio $S_{\mathrm{mag}} / R_{\mathrm{mag}}$ is maximized.

From a measured value $\operatorname{LOD}(1 \mathrm{~Hz})=292 \mathrm{pT} / \sqrt{\mathrm{Hz}}$ at $B_{\text {bias }}=+0.65 \mathrm{mT}$ the relative magnetic loss 
factor can be determined to $\tan \delta_{\mathrm{mag}} / \mu_{\mathrm{r}}^{\prime}=1.6 \cdot 10^{-4}$ $\left(\tan \delta_{\mathrm{mag}} / \mu_{\mathrm{r}}^{\prime}=2.9 \cdot 10^{-4}\right.$ at $\left.B_{\text {bias }}=-0.65 \mathrm{mT}\right)$. Due to the dependence of the magnetic properties on e.g. the material composition, thickness, magnetic domain configuration, shape, etc. and also due to their interdependencies it is difficult to compare the determined value with other values from the literature. However, values reported in [70] are at least in the same order of magnitude even though the investigated samples were measured at cryogenic temperatures. For typical parameters of the sensor under investigation, Fig. 10 depicts resulting limits of detection at a frequency of $1 \mathrm{~Hz}$ for various relative magnetic loss factors. Because the LOD is proportional to the square root of this loss factor, $\tan \delta_{\mathrm{mag}} / \mu_{\mathrm{r}}^{\prime}$ needs to be decreased by two orders of magnitude in order to improve the LOD by a factor of ten.

\section{CONCLUSION}

In this paper, a cantilever-type magnetoelastic resonant sensor, representative for other kinds of magnetoelastic resonators, has been investigated. Such sensors for the detection of low-frequency and low-amplitude magnetic fields utilize the $\Delta \mathrm{E}$ effect which leads to a magnetically induced resonance detuning. For the detection of the resonator's detuning, the sensor is preferably driven by an electrical excitation signal which, in turn, is then phase modulated by the magnetic measurement signal. Based on the dynamics of resonant mechanical structures an expression for the overall phase sensitivity has been derived. Such sensors exhibit several loss mechanisms that lead to random vibrations of the structure (thermal-mechanical noise) as well as to random agitation of the charge carriers flowing through the sensor (thermalelectrical noise). The phase noise resulting from these thermal noise sources can not only be predicted accurately but also decreased easily by increasing the excitation amplitude (additive noise). However, it has been shown that losses appearing in the sensor's magnetic material due to domain wall actions clearly generate additional flicker phase noise that can not be decreased by increasing the excitation amplitude (parametric noise). Based on the fluctuation-dissipation theorem indicating random fluctuations of the magnetization, an analytical expression for the magnetically induced phase noise could be derived. With this result, not only the fundamental LOD due to thermal vibrations of the mechanical structure but also the LOD for sensors impaired by magnetically induced phase noise could be described. In particular, in the latter case, the LOD does not depend on the sensitivity but is solely determined by the dynamic loss properties of the magnetic layer, at least if the magnetic sensitivity is high enough such that thermal noise sources are negligible. Hence, instead of the sensitivity, the magnetic losses, represented by the material's effective complex permeability, should be considered as the most important parameter for the further improvement of such sensors. This implication is not only valid for magnetoelastic cantilevers but also applies to any type of magnetoelastic resonator.

Please note that all statements made in this paper refer to magnetic fields oriented along the long axis of the cantilever as depicted in Fig. 1. If the low-amplitude magnetic measurement signal $B_{\mathrm{x}}(t)$ is applied at a different angle, only the sensitivity decreases cosinusoidally while all noise contributions remain unchanged. Thus, independently of the dominant noise source, the LOD worsens by the same factor the sensitivity is decreased. Contrary, if the ambient magnetic bias flux density $B_{\text {bias }}$ is applied at a different angle, the sensor's magnetic state might be altered significantly. Due to the tight relation between the magnetic state and the magnetic losses, not only the magnetic sensitivity but also the noise contributions will change (with exception of the thermal-electrical noise caused by the dielectric losses of the piezoelectric material). As long as the overall noise floor is dominated by random fluctuations of the magnetization, the LOD is still exclusively determined by the relative magnetic loss factor (Eq. (54)).

\section{ACKNOWLEDGMENT}

This work was supported (1) by the German Research Foundation (Deutsche Forschungsgemeinschaft, DFG) through the Collaborative Research Centre CRC 1261 Magnetoelectric Sensors: From Composite Materials to Biomagnetic Diagnostics, (2) by the ANR Programme d'Investissement d'Avenir (PIA) under the Oscillator IMP project (project 11-EQPX0033) and the FIRST-TF network (project 10-LABX-0048), and (3) by grants from the Région Bourgogne Franche-Comté intended to support the PIA.

\section{REFERENCES}

[1] R. Brendel, C. El Hassani, M. Brunet, and E. Robert, "Influence of magnetic field on quartz crystal oscillators," in Proceedings of the 43rd Annual Symposium on Frequency Control, 31. May - 2. June 1989, Denver, Colorado, USA, 1989, pp. 268-274.

[2] R. Brendel, "Influence of a magnetic field on quartz crystal resonators," IEEE Transactions on Ultrasonics, Ferroelectrics and Frequency Control, vol. 43, no. 5, pp. 818-831, Sept. 1996.

[3] S. Hanna, "Magnetic Field Sensors Based on SAW Propagation in Magnetic Films," IEEE Transactions on Ultrasonics, Ferroelectrics and Frequency Control, vol. 34, no. 2, pp. 191-194, Mar. 1987.

[4] W. Li, P. Dhagat, and A. Jander, "Surface Acoustic Wave Magnetic Sensor using Galfenol Thin Film,' IEEE Transactions on Magnetics, vol. 48, no. 11, pp. 4100-4102, Nov. 2012.

[5] M. Elhosni, S. Petit-Watelot, M. Hehn, S. Hage-Ali, K. A. Aissa, D. Lacour, A. Talbi, and O. Elmazria, "Experimental Study of Multilayer Piezo-magnetic SAW Delay Line for Magnetic Sensor," Procedia Engineering, vol. 120, pp. 870-873, 2015.

[6] A. Kittmann, P. Durdaut, S. Zabel, J. Reermann, J. Schmalz, B. Spetzler, D. Meyners, N. X. Sun, J. McCord, M. Gerken, G. Schmidt, M. Höft, R. Knöchel, F. Faupel, and E. Quandt, "Wide Band Low Noise Love Wave Magnetic Field Sensor System," Scientific Reports, vol. 8, no. 1, pp. 278-287, Jan. 2018.

[7] A. Mazzamurro, A. Talbi, Y. Dusch, O. Elmazria, P. Pernod, O. B. Matar, and N. Tiercelin, "Highly Sensitive Surface Acoustic Wave Magnetic Field Sensor Using Multilayered TbCo2/FeCo Thin Film," Proceedings, vol. 2, no. 13, p. 902, Nov. 2018.

[8] Wen, Yana, Xufeng, Yong, and Zhaofu, "Magnetostrictive effect in micro-dotted FeCo film coated surface acoustic wave devices," Smart Materials and Structures, vol. 27, no. 10, p. 105040, Oct. 2018.

[9] V. Schell, C. Müller, P. Durdaut, A. Kittmann, L. Thormählen, F. Lofink, D. Meyners, M. Höft, J. McCord, and E. Quandt, "Magnetic anisotropy controlled FeCoSiB thin films for surface acoustic wave magnetic field sensors," Applied Physics Letters, vol. 116, no. 7, pp. 073503 1-5, Feb. 2020.

[10] N. Yoshizawa, I. Yamamoto, and Y. Shimada, "Magnetic field sensing by an electrostrictive/magnetostrictive composite resonator," IEEE Transactions on Magnetics, vol. 41, no. 11, pp. 4359-4361, Nov. 2005. 
[11] T. Nan, Y. Hui, M. Rinaldi, and N. X. Sun, "Self-Biased $215 \mathrm{MHz}$ Magnetoelectric NEMS Resonator for Ultra-Sensitive DC Magnetic Field Detection," Scientific Reports, vol. 3, no. 1, p. 1985, Dec. 2013.

[12] J. Kiser, P. Finkel, J. Gao, C. Dolabdjian, J. Li, and D. Viehland, "Stress reconfigurable tunable magnetoelectric resonators as magnetic sensors," Applied Physics Letters, vol. 102, no. 4, pp. 042909 1-4, Jan. 2013.

[13] Y. Hui, T. Nan, N. X. Sun, and M. Rinaldi, "High Resolution Magnetometer Based on a High Frequency Magnetoelectric MEMS-CMOS Oscillator," Journal of Microelectromechanical Systems, vol. 24, no. 1 , pp. 134-143, Feb. 2015.

[14] L. Bian, Y. Wen, P. Li, Y. Wu, X. Zhang, and M. Li, "Magnetostrictive stress induced frequency shift in resonator for magnetic field sensor," Sensors and Actuators A: Physical, vol. 247, pp. 453-458, Aug. 2016.

[15] M. Li, A. Matyushov, C. Dong, H. Chen, H. Lin, T. Nan, Z. Qian, M. Rinaldi, Y. Lin, and N. X. Sun, "Ultra-sensitive NEMS magnetoelectric sensor for picotesla DC magnetic field detection," Applied Physics Letters, vol. 110, no. 14, pp. 143510 1-4, Apr. 2017.

[16] M. Staruch, M.-T. Yang, J. F. Li, C. Dolabdjian, D. Viehland, and P. Finkel, "Frequency reconfigurable phase modulated magnetoelectric sensors using $\Delta \mathrm{E}$ effect," Applied Physics Letters, vol. 111, no. 3, pp. $0329051-4$, July 2017.

[17] S. P. Bennett, J. W. Baldwin, M. Staruch, B. R. Matis, J. LaComb, O. M. J. van 't Erve, K. Bussmann, M. Metzler, N. Gottron, W. Zappone, R. LaComb, and P. Finkel, "Magnetic field response of doubly clamped magnetoelectric microelectromechanical AlN-FeCo resonators," Applied Physics Letters, vol. 111, no. 25, pp. 252903 1-5, Dec. 2017.

[18] L. Bian, Y. Wen, Y. Wu, P. Li, Z. Wu, Y. Jia, and Z. Zhu, "A Resonant Magnetic Field Sensor With High Quality Factor Based on Quartz Crystal Resonator and Magnetostrictive Stress Coupling," IEEE Transactions on Electron Devices, vol. 65, no. 6, pp. 2585-2591, June 2018.

[19] R. Osiander, S. A. Ecelberger, R. B. Givens, D. K. Wickenden, J. C. Murphy, and T. J. Kistenmacher, "A microelectromechanical-based magnetostrictive magnetometer," Applied Physics Letters, vol. 69, no. 19, pp. 2930-2931, Nov. 1996.

[20] B. Gojdka, R. Jahns, K. Meurisch, H. Greve, R. Adelung, E. Quandt, R. Knöchel, and F. Faupel, "Fully integrable magnetic field sensor based on delta-E effect," Applied Physics Letters, vol. 99, no. 22, pp. 223502 1-3, Nov. 2011

[21] R. Jahns, S. Zabel, S. Marauska, B. Gojdka, B. Wagner, R. Knöchel, R. Adelung, and F. Faupel, "Microelectromechanical magnetic field sensor based on $\Delta \mathrm{E}$ effect," Applied Physics Letters, vol. 105, no. 5, pp. 052414 1-3, Aug. 2014.

[22] S. Zabel, C. Kirchhof, E. Yarar, D. Meyners, E. Quandt, and F. Faupel, "Phase modulated magnetoelectric delta-E effect sensor for sub-nano tesla magnetic fields," Applied Physics Letters, vol. 107, no. 15, pp. 152402 1-4, Oct. 2015

[23] Y. Park, E. Lee, and T. Kouh, "Field-Dependent Resonant Behavior of Thin Nickel Film-Coated Microcantilever," Micromachines, vol. 8, no. 4, p. 109, Apr. 2017.

[24] P. Durdaut, S. Salzer, J. Reermann, P. Hayes, D. Meyners, E. Quandt, G. Schmidt, R. Knöchel, and M. Höft, "Thermal-Mechanical Noise in Resonant Thin-Film Magnetoelectric Sensors," IEEE Sensors Journal, vol. 17, no. 8, pp. 2338-2348, Apr. 2017.

[25] P. Durdaut, V. Penner, C. Kirchhof, E. Quandt, R. Knöchel, and M. Höft, "Noise of a JFET Charge Amplifier for Piezoelectric Sensors," IEEE Sensors Journal, vol. 17, no. 22, pp. 7364-7371, Nov. 2017.

[26] P. Durdaut, J. Reermann, S. Zabel, C. Kirchhof, E. Quandt, F. Faupel, G. Schmidt, R. Knöchel, and M. Höft, "Modeling and Analysis of Noise Sources for Thin-Film Magnetoelectric Sensors Based on the DeltaE Effect," IEEE Transactions on Instrumentation and Measurement, vol. 66, no. 10, pp. 2771-2779, Oct. 2017.

[27] P. Durdaut, A. Kittmann, E. Rubiola, J.-M. Friedt, E. Quandt, R. Knöchel, and M. Höft, "Noise Analysis and Comparison of Phaseand Frequency-Detecting Readout Systems: Application to SAW Delay Line Magnetic Field Sensor," IEEE Sensors Journal, vol. 19, no. 18, pp. 8000-8008, Sept. 2019.

[28] P. Durdaut, A. Kittmann, A. Bahr, E. Quandt, R. Knöchel, and M. Höft, "Oscillator Phase Noise Suppression in Surface Acoustic Wave Sensor Systems," IEEE Sensors Journal, vol. 18, no. 12, pp. 4975-4980, June 2018.

[29] L. Daniel and O. Hubert, "An analytical model for the $\Delta \mathrm{E}$ effect in magnetic materials," The European Physical Journal Applied Physics, vol. 45, no. 3, p. 31101, Mar. 2009.

[30] B. D. Cullity, Introduction to Magnetic Materials. Hoboken, New Jersey, USA: IEEE Press, Wiley, 2009.
[31] A. E. Clark, J. B. Restorff, M. Wun-Fogle, and J. F. Lindberg, "Magnetoelastic coupling and $\Delta \mathrm{E}$ effect in $\mathrm{Tb}_{x} \mathrm{Dy}_{1-x}$ single crystals," Journal of Applied Physics, vol. 73, no. 10, pp. 6150-6152, May 1993.

[32] A. Ludwig and E. Quandt, "Optimization of the $\Delta \mathrm{E}$ effect in thin films and multilayers by magnetic field annealing," IEEE Transactions on Magnetics, vol. 38, no. 5, pp. 2829-2831, Sept. 2002.

[33] C. Dong, M. Li, X. Liang, H. Chen, H. Zhou, X. Wang, Y. Gao, M. E. McConney, J. G. Jones, G. J. Brown, B. M. Howe, and N. X. Sun, "Characterization of magnetomechanical properties in $\mathrm{FeGaB}$ thin films," Applied Physics Letters, vol. 113, no. 26, pp. 262401 1-5, Dec. 2018.

[34] E. Yarar, V. Hrkac, C. Zamponi, A. Piorra, L. Kienle, and E. Quandt, "Low temperature aluminum nitride thin films for sensory applications," AIP Advances, vol. 6, no. 7, pp. 075115 1-11, July 2016.

[35] S. Zabel, J. Reermann, S. Fichtner, C. Kirchhof, E. Quandt, B. Wagner, G. Schmidt, and F. Faupel, "Multimode delta-E effect magnetic field sensors with adapted electrodes," Applied Physics Letters, vol. 108, no. 22, pp. 222401 1-4, May 2016.

[36] A. Hubert and R. Schäfer, Magnetic Domains: The Analysis of Magnetic Microstructures. Berlin, Germany: Springer Science+Business Media, 2009.

[37] N. O. Urs, I. Teliban, A. Piorra, R. Knöchel, E. Quandt, and J. McCord, "Origin of hysteretic magnetoelastic behavior in magnetoelectric 2-2 composites," Applied Physics Letters, vol. 105, no. 20, pp. 202406 1-5, Nov. 2014.

[38] N. O. Urs, E. Golubeva, V. Röbisch, S. Toxvaerd, S. Deldar, R. Knöchel, M. Höft, E. Quandt, D. Meyners, and J. McCord, "Direct Link between Specific Magnetic Domain Activities and Magnetic Noise in Modulated Magnetoelectric Sensors," Physical Review Applied, vol. 13, no. 2, pp. 024018 1-10, Feb. 2020

[39] F. Brailsford, "Domain-wall energy and hysteresis loss in ferromagnetic material," Proceedings of the Institution of Electrical Engineers, vol. 117 , no. 5, pp. 1052-1055, 1970

[40] W. Campbell, Introduction to Geomagnetic Fields. Cambridge, United Kingdom: Cambridge University Press, 2003.

[41] D. Zhang, Z. Pan, H. Zhou, and W. Zhang, "Magnetic sensor based on giant magneto-impedance effect using the self-regulating technology on the bias magnetic field," Sensors and Actuators, A: Physical, vol. 249, pp. 225-230, 2016.

[42] H. Zhou, Z. M. Pan, and D. S. Zhang, "Operating Point Self-Regulator for Giant Magneto-Impedance Magnetic Sensor," Sensors, vol. 17, no. 5, p. 1103, May 2017.

[43] E. Lage, N. O. Urs, V. Röbisch, I. Teliban, R. Knöchel, D. Meyners, J. McCord, and E. Quandt, "Magnetic domain control and voltage response of exchange biased magnetoelectric composites," Applied Physics Letters, vol. 104, no. 13, pp. 132405 1-4, Mar. 2014.

[44] C. V. Heer, Statistical Mechanics, Kinetic Theory, and Stochastic Processes. New York City, New York, USA: Academic Press, 1972.

[45] K. Kobayashi, H. Yamada, and K. Matsushige, "Frequency noise in frequency modulation atomic force microscopy," Review of Scientific Instruments, vol. 80, no. 4, pp. 043708 1-8, Apr. 2009.

[46] P. Durdaut, M. Höft, J.-M. Friedt, and E. Rubiola, "Equivalence of OpenLoop and Closed-Loop Operation of SAW Resonators and Delay Lines," Sensors, vol. 19, no. 185, p. 185, Jan. 2019.

[47] J. Mertz, O. Marti, and J. Mlynek, "Regulation of a microcantilever response by force feedback," Applied Physics Letters, vol. 62, no. 19, pp. 2344-2346, May 1993.

[48] J. Reermann, S. Zabel, C. Kirchhof, E. Quandt, F. Faupel, and G. Schmidt, "Adaptive Readout Schemes for Thin-Film Magnetoelectric Sensors Based on the delta-E Effect," IEEE Sensors Journal, vol. 16 , no. 12 , pp. 4891-4900, June 2016.

[49] A. Bloch, "Electromechanical analogies and their use for the analysis of mechanical and electromechanical systems," Journal of the Institution of Electrical Engineers - Part I: General, vol. 92, no. 52, pp. 157-169, Apr. 1945.

[50] C. Kirchhof, M. Krantz, I. Teliban, R. Jahns, S. Marauska, B. Wagner, R. Knöchel, M. Gerken, D. Meyners, and E. Quandt, "Giant magnetoelectric effect in vacuum," Applied Physics Letters, vol. 102, no. 23, p. 232905, June 2013.

[51] M. Weinberg, R. Candler, S. Chandorkar, J. Varsanik, T. Kenny, and A. Duwel, "Energy loss in MEMS resonators and the impact on inertial and RF devices," in Proceedings of the 15th International Conference on Solid-State Sensors, Actuators and Microsystems, 21. June - 25. June 2009, vol. 1, Denver, Colorado, USA, June 2009, pp. 688-695.

[52] E. Villari, "Ueber die Aenderungen des magnetischen Moments, welche der Zug und das Hindurchleiten eines galvanischen Stroms in einem 
Stabe von Stahl oder Eisen hervorbringen," Annalen der Physik und Chemie, vol. 202, no. 9, pp. 87-122, 1865.

[53] G. Bertotti, "General-Properties of Power Losses in Soft Ferromagnetic Materials," IEEE Transactions on Magnetics, vol. 24, no. 1, pp. 621630, Jan. 1988.

[54] J. Goodenough, "Summary of losses in magnetic materials," IEEE Transactions on Magnetics, vol. 38, no. 5, pp. 3398-3408, Sept. 2002.

[55] E. Yarar, V. Hrkac, C. Zamponi, A. Piorra, L. Kienle, and E. Quandt, "Low temperature aluminum nitride thin films for sensory applications," AIP Advances, vol. 6, no. 7, p. 075115, July 2016.

[56] S. Fichtner, N. Wolff, G. Krishnamurthy, A. Petraru, S. Bohse, F. Lofink, S. Chemnitz, H. Kohlstedt, L. Kienle, and B. Wagner, "Identifying and overcoming the interface originating c-axis instability in highly Sc enhanced AlN for piezoelectric micro-electromechanical systems," Journal of Applied Physics, vol. 122, no. 3, pp. 035301 1-7, July 2017.

[57] A. Piorra, R. Jahns, I. Teliban, J. L. Gugat, M. Gerken, R. Knöchel, and E. Quandt, "Magnetoelectric thin film composites with interdigital electrodes," Applied Physics Letters, vol. 103, no. 3, pp. 032902 1-5, July 2013

[58] T. B. Jones and N. G. Nenadic, Electromechanics and MEMS. Cambridge, United Kingdom: Cambridge University Press Textbooks, 2013.

[59] R. D. Grober, J. Acimovic, J. Schuck, D. Hessman, P. J. Kindlemann, J. Hespanha, A. S. Morse, K. Karrai, I. Tiemann, and S. Manus, "Fundamental limits to force detection using quartz tuning forks," Review of Scientific Instruments, vol. 71, no. 7, pp. 2776-2780, July 2000.

[60] A. Arnau, J. V. García, Y. Jimenez, V. Ferrari, and M. Ferrari, "Improved electronic interfaces for AT -cut quartz crystal microbalance sensors under variable damping and parallel capacitance conditions," Review of Scientific Instruments, vol. 79, no. 7, pp. 075110 1-12, July 2008.

[61] Z. Hu, J. Hedley, N. Keegan, J. Spoors, B. Gallacher, and C. McNeil, "One-Port Electronic Detection Strategies for Improving Sensitivity in Piezoelectric Resonant Sensor Measurements," Sensors, vol. 16, no. 11, p. 1781 , Oct. 2016.

[62] A. G. Fowler, M. B. Coskun, and S. O. R. Moheimani, "Q control of a microfabricated piezoelectric cantilever with on-chip feedthrough cancellation," in Proceedings 2017 IEEE Conference on Control Technology and Applications, 27.-30. Aug. 2017, Kohala Coast, Hawaii, USA, Aug. 2017, pp. 123-128.

[63] A. L. Lance, W. D. Seal, and F. Labaar, "Phase Noise and AM Noise Measurements in the Frequency Domain," in Infrared and Millimeter Waves. Orlando, Florida, USA: Academic Press, Inc., 1984, vol. 11.

[64] P. D. Welch, "The Use of Fast Fourier Transform for the Estimation of Power Spectra: A Method Based on Time Averaging Over Short, Modified Periodograms," IEEE Transactions on Audio and Electroacoustics, vol. 15 , no. 2, pp. 70-73, 1967.

[65] E. Rubiola, Phase Noise and Frequency Stability in Oscillators. Cambridge, UK: Cambridge University Press, 2009.

[66] R. Boudot and E. Rubiola, "Phase noise in RF and microwave amplifiers," IEEE Transactions on Ultrasonics, Ferroelectrics and Frequency Control, vol. 59, no. 12, pp. 2613-2624, Dec. 2012.

[67] H. T. Hardner, M. B. Weissman, M. B. Salamon, and S. S. P. Parkin, "Fluctuation-dissipation relation for giant magnetoresistive 1/f noise," Physical Review B, vol. 48, no. 21, pp. 16156-16159, Dec. 1993.

[68] R. J. M. van de Veerdonk, P. J. L. Beliën, K. M. Schep, J. C. S. Kools, M. C. de Nooijer, M. A. M. Gijs, R. Coehoorn, and W. J. M. de Jonge, "1/f noise in anisotropic and giant magnetoresistive elements," Journal of Applied Physics, vol. 82, no. 12, pp. 6152-6164, Dec. 1997.

[69] S. Ingvarsson, G. Xiao, S. S. P. Parkin, W. J. Gallagher, G. Grinstein, and R. H. Koch, "Low-Frequency Magnetic Noise in Micron-Scale Magnetic Tunnel Junctions," Physical Review Letters, vol. 85, no. 15, pp. 32893292 , Oct. 2000.

[70] G. Durin, P. Falferi, M. Cerdonio, G. A. Prodi, and S. Vitale, "Low temperature properties of soft magnetic materials: Magnetic viscosity and 1/f thermal noise," Journal of Applied Physics, vol. 73, no. 10, pp. 5363-5365, 1993.

[71] J. Briaire, "1/f Noise in Permalloy," Ph.D. dissertation, Eindhoven University of Technology, 2000.

[72] S.-K. Lee and M. V. Romalis, "Calculation of magnetic field noise from high-permeability magnetic shields and conducting objects with simple geometry," Journal of Applied Physics, vol. 103, no. 8, pp. 084904 1-10, Apr. 2008.

[73] W. Jayasekara, J. Bain, and M. Kryder, "High frequency initial permeability of NiFe and FeAlN," IEEE Transactions on Magnetics, vol. 34, no. 4, pp. 1438-1440, July 1998.

[74] L. G. C. Melo, D. Ménard, A. Yelon, L. Ding, S. Saez, and C. Dolabdjian, "Optimization of the magnetic noise and sensitivity of giant magnetoimpedance sensors," Journal of Applied Physics, vol. 103, no. 3, pp. 033903 1-6, Feb. 2008. [Online]. Available: http://aip.scitation.org/doi/10.1063/1.2837106

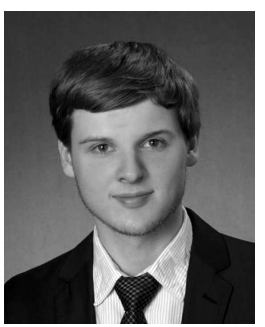

Phillip Durdaut received a B.Eng. degree in Information and Electrical Engineering from the University of Applied Sciences Hamburg, Germany, and an M.Sc. degree in Electrical Engineering and Information Technology from Kiel University, Germany, in 2013 and 2015, respectively. Since 2015, he is a Research Associate with the Chair of Microwave Engineering at Kiel University, Germany, where he obtained the Dr.-Ing. degree in 2019. His main research interests include the analysis and the development of low-noise magnetic field sensor systems based on microelectromechanical resonators and surface acoustic wave devices.

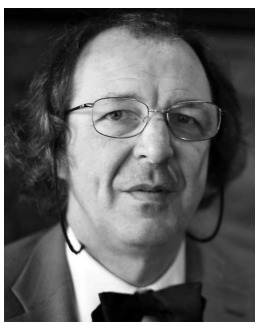

Enrico Rubiola received the M.S. degree in electronics engineering from the Politecnico di Torino, Turin, Italy, in 1983, the Ph.D. degree in metrology from the Italian Ministry of Scientific Research, Rome, Italy, in 1989, and the D.Sc. degree in time and frequency metrology from the University of Franche-Comté (UFC), Besançon, France, in 1999. He has been a Researcher with the Politecnico di Torino, a Professor with the University of Parma, Parma, Italy, and Université Henri Poincaré, Nancy, France, and a Guest Scientist with the NASA Jet Propulsion Laboratory, Pasadena, CA, USA. He has been a Professor with UFC and a Scientist with the Centre National de la Recherche Scientifique, Franche Comté Électronique Mécanique Thermique et Optique-Sciences et Technologies Institute, Besançon, since 2005, where he currently serves as the Deputy Director of the Time and Frequency Department. He has also investigated various topics of electronics and metrology, namely, navigation systems, time and frequency comparisons, atomic frequency standards, and gravity. $\mathrm{He}$ is the PI of the Oscillator IMP project, a platform for the measurement of short-term frequency stability and spectral purity. His current research interests include precision electronics, phase-noise, amplitude-noise, frequency stability and synthesis, and low-noise oscillators from the low RF region to optics.

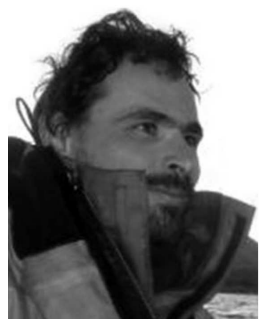

Jean-Michel Friedt received the Ph.D. degree from the University of Franche-Comté, Besançon, France, in 2000 and the Habilitation degree from the Time and Frequency Department, FEMTO-ST, Besançon, France, in 2010,In 2004, he joined the group of S. Ballandras, FEMTO-ST, for the development of passive sensors interrogated through a wireless link. He was a Postdoctoral Fellow with IMEC, Leuven, Belgium, where he was involved in acoustic and optical biosensors for characterizing organic thin film properties. He has been an Engineer with the SENSeOR company, Valbonne, France, since its creation in 2006, hosted by the Time and Frequency Department, FEMTO-ST. He has been an Associate Professor with the University of Franche-Comté, Besançon, since 2014, where he was teaching radio frequency and embedded electronics. His research interests include scanning probe microscopy, passive radio frequency sensors, the associated radarlike electronics implemented as software-defined radio, and the associated digital signal processing techniques. 


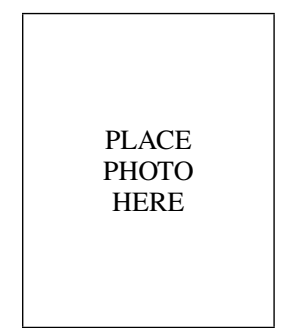

Cai Müller received the M.Sc. degree in materials science and engineering from the University of Kiel, Kiel, Germany, in 2015, where he is currently pursuing the Dr.-Ing. degree with the Chair of Nanoscale Magnetic Materials - Magnetic Domains. His research interests include magnetic domains and magnetization dynamics in magnetic thin films.

Benjamin Spetzler ...
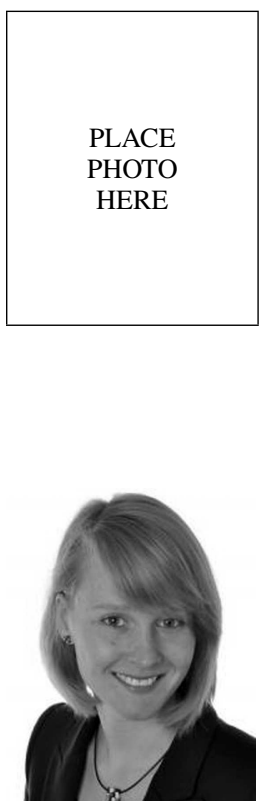

Christine Kirchhof ...

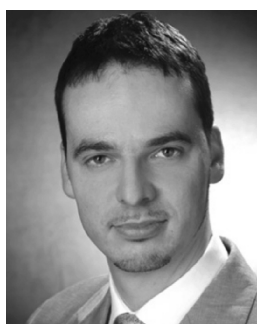

Dirk Meyners received the Diploma and Ph.D degrees from Bielefeld University, Germany, in 2001 and 2006, respectively. After being a Postdoctoral Researcher with the Center of Advanced European Studies and Research, Bonn, Germany, he joined the Inorganic Functional Materials Group, Kiel University, Germany, in 2007. Since 2008, he has been the Scientific Director of the Kiel Nanolaboratory. His current research interests include the magnetism of thin films and microstructures, and the investigation of sensors based on phenomena, such as tunneling magnetoresistance, magnetostrictive, and magnetoelectric effects.

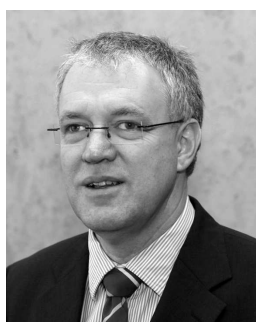

Eckhard Quandt received the Diploma and Dr.-Ing. degrees in physics from the Technische Universität Berlin, Germany, in 1986 and 1990, respectively. He is currently a Professor with Kiel University, Kiel, Germany, where he is also the Director with the Institute for Materials Science. He is the spokesperson of the DFG CRC 1261 Magnetoelectric Sensors: From Composite Materials to Biomagnetic Diagnostics, and a member of Acatech, National Academy of Science and Engineering. His scientific focus is material research on smart materials and multiferroics and the development of sensors and actuators for microelectromechanical systems and nanoelectromechanical systems using such materials.

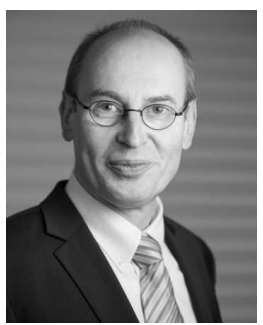

Franz Faupel received his Ph.D. in physics from the University of Göttingen in 1985. From 1987 to 1988, he was postdoctoral fellow at the IBM Th. J. Watson Research Center in Yorktown Heights, New York and got his habilitation from the University of Göttingen in 1992. Since 1994, he has been a full professor and holds the Chair for Multicomponent Materials within the Faculty of Engineering at Kiel University, where he is also a faculty member in the Physics Department. Faupel is Chairman of the North German Initiative Nanotechnology and Principal Editor of the Journal of Materials Research. He also served in the editorial boards of Applied Physics Letters, the Journal of Applied Physics, and other journals. Among various additional duties, he is member of MinervaWeizmann Committee of the Max Planck Society, and from 2008 to 2010, he was Dean of the Faculty of Engineering. Faupel published more than 300 peer reviewed papers and is listed in the Web of Science with an h-index of 40. His research interests include functional nanocomposites, magnetoelectric sensors, diffusion, metallic glasses, metal-polymer interfaces and plasma deposition.

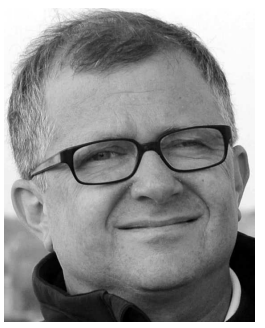

Jeffrey McCord received the Dipl.-Ing. and Ph.D. degrees in materials science and engineering from the University of Erlangen-Nürnberg, Germany. From 1997 to 2001, he was with IBM Storage Division, San Jose, USA, developing magnetic recording head materials. From 2002 to 2009, he was a Research Scientist and a Group Leader with the Leibniz Institute for Solid State Physics and Materials Science, Dresden, Germany, until he took over a position as the Head of Department Nanomagnetism at the Helmholtz-Center Dresden-Rossendorf, Dresden. $\mathrm{He}$ is Co-Founder, Partner, and served as Director of Evico Magnetics $\mathrm{GmbH}$, Germany. He joined the University of Kiel as a Heisenberg-Professor for materials science in 2011. His primary research focuses on magnetic domains and magnetic materials. The topics of research range from fundamental research to engineering materials concentrating on magnetization dynamics, exchange bias, magnetic thin films, and nanomagnetism.

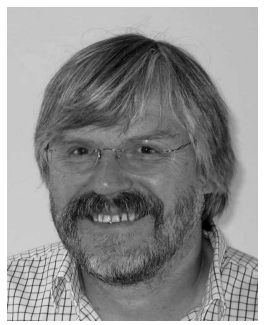

Reinhard Knöchel received a Dipl.-Ing. degree in electrical engineering and a Dr.-Ing. degree from the Technical University, Braunschweig, Germany, in 1975 and 1980, respectively. From 1980 to 1986, he was a Principal Scientist with the Philips Research Laboratory, Hamburg, Germany. In 1986, he joined the Hamburg University of Technology, where he was a Full Professor of Microwave Electronics until 1993. Between 1993 and 2013, he held the Chair in Microwave Engineering Kiel University, Kiel, Germany. His research interests include magnetic field sensors, active and passive microwave components, ultrawideband technology, microwave measurement techniques, industrial microwave sensors and radar. 


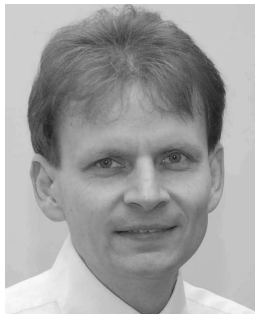

Michael Höft received a Dipl.-Ing. degree in electrical engineering and a Dr.-Ing. degree from the Hamburg University of Technology, Hamburg, Germany, in 1997 and 2002, respectively. From 2002 to 2013, he was with the Communications Laboratory, European Technology Center, Panasonic Industrial Devices Europe GmbH, Lüneburg, Germany. He was a Research Engineer and then a Team Leader, where he was involved in the research and development of microwave circuitry and components, particularly filters for cellular radio communications. From 2010 to 2013, he was also a Group Leader for the research and development of sensor and network devices. Since 2013, he has been a Full Professor at Kiel University, Kiel, Germany, in the Faculty of Engineering, where he is the Head of the Chair of Microwave Engineering of the Institute of Electrical Engineering and Information Technology. His research interests include active and passive microwave components, (sub-) millimeter-wave quasi-optical techniques and circuitry, microwave and field measurement techniques, microwave filters, microwave sensors and magnetic field sensors. $\mathrm{He}$ is a member of the European Microwave Association, the Association of German Engineers and a member of the German Institute of Electrical Engineers. 